OPEN ACCESS

Edited by:

Stephen J. Pandol,

Cedars-Sinai Medical Center,

United States

Reviewed by:

Nick Spencer,

Flinders University, Australia Marcello Costa,

Flinders University, Australia Shin-Ichiro Karaki,

University of Shizuoka, Japan

*Correspondence:

Jan D. Huizinga

huizinga@mcmaster.ca

tThese authors share first authorship

¥These authors share senior authorship

Specialty section

This article was submitted to

Gastrointestinal Sciences,

a section of the journal

Frontiers in Physiology

Received: 03 November 2019

Accepted: 30 January 2020

Published: 18 February 2020

Citation:

Tan W, Lee G, Chen J-H and

Huizinga JD (2020) Relationships

Between Distention-, Butyrate-

and Pellet-Induced Stimulation

of Peristalsis in the Mouse Colon.

Front. Physiol. 11:109

doi: 10.3389/fphys.2020.00109

\section{Relationships Between Distention-, Butyrate- and Pellet-Induced Stimulation of Peristalsis in the Mouse Colon}

\author{
Wei Tan ${ }^{1,2 t}$, Grace Lee ${ }^{2 t}$, Ji-Hong Chen ${ }^{2 \ddagger}$ and Jan D. Huizinga ${ }^{2 * \neq}$ \\ ${ }^{1}$ Department of Gastroenterology, Renmin Hospital of Wuhan University, Wuhan, China, ${ }^{2}$ Department of Medicine, Division \\ of Gastroenterology, Farncombe Family Digestive Health Research Institute, McMaster University, Hamilton, ON, Canada
}

Background/Aims: Luminal factors such as short-chain fatty acids are increasingly recognized for playing a regulatory role in peristaltic activity. Our objective was to understand the roles of butyrate and propionate in regulating peristaltic activity in relation to distention-induced activities.

Methods: Butyrate and propionate were perfused intraluminally under varying intraluminal pressures in murine colons bathed in Krebs solution. We used video recording and spatiotemporal maps to examine peristalsis induced by the intrinsic rhythmic colonic motor complex $(\mathrm{CMC})$ as well as pellet-induced peristaltic reflex movements.

Results: The CMC showed several configurations at different levels of excitation, culminating in long distance contractions (LDCs) which possess a triangular shape in murine colon spatiotemporal maps. Butyrate increased the frequency of CMCs but was a much weaker stimulus than distention and only contributed to significant changes under low distention. Propionate inhibited CMCs by decreasing either their amplitudes or frequencies, but only in low distention conditions. Butyrate did not consistently counteract propionate-induced inhibition likely due to the multiple and distinct mechanisms of action for these signaling molecules in the lumen. Pellet movement occurred through ongoing CMCs as well as pellet induced peristaltic reflex movements and butyrate augmented both types of peristaltic motor patterns to decrease the amount of time required to expel each pellet.

Conclusions: Butyrate is effective in promoting peristalsis, but only when the level of colonic activity is low such as under conditions of low intraluminal pressure. This suggests that it may play a significant role in patients with poor fiber intake, where there is low mechanical stimulation in the lumen.

Keywords: butyrate, propionate, peristalsis, peristaltic reflex, colonic motility

\section{INTRODUCTION}

Colonic motility is dependent on stimuli, and colonic distention is a major stimulus for the initiation of motor patterns. Recently, high-resolution spatio-temporal mapping has given us more insight into motor patterns in the mouse colon (Roberts et al., 2007, 2008; Vincent et al., 2018; Beck et al., 2019) the rabbit colon (Dinning et al., 2014; Chen et al., 2016) and the human 
colon (Dinning, 2016; Chen et al., 2017, 2018; Corsetti et al., 2017). Despite the fact that luminal stimulation is critical for normal colonic motility, the role of luminal factors in controling or modifying motility has not been extensively studied. When digested, content leaves the small intestine and enters the colon. The content causes distension of the colon and/or increases in luminal pressure that evokes motor activity; at the same time, microbial fermentation produces metabolites that might activate [e.g., butyrate (Hurst et al., 2014), 5-HT (Fukumoto et al., 2003)] or inhibit [e.g., propionate (Hurst et al., 2014)] motility. Hence under normal conditions, the colon appears to find a balance between different stimuli to generate motor patterns that facilitate digestion, absorption, and microbiota homeostasis. Consequently, poor colonic motility may be caused by an imbalance in these factors. In support of this hypothesis, we recently found that abnormal motor patterns in germ-free mice can be reversed to normal by luminal butyrate (Vincent et al., 2018). Our goal for the present study was to understand how butyrate, a natural short chain fatty acid (SCFA) in the colon, modulates peristaltic activity by evaluating its effects in relation to both distention and propionate, another natural SCFA that appears to have effects opposite to that of butyrate. Knowledge of butyrate's effects could indicate whether its potential roles can be leveraged as an intraluminal prokinetic in motility disorders.

In order to fully understand the effect of SCFAs on the generation of peristaltic motor patterns, we have to distinguish two types of peristalsis. Peristalsis can occur through the generation of a neurally induced rhythmic motor pattern, the colonic motor complex (CMC) (Corsetti et al., 2019), previously referred to in the literature as the colonic migrating motor complex in the mouse colon (Fida et al., 1997; Bayguinov et al., 2010) or the peristaltic wave (Smith et al., 2003) or cyclic motor complex in the guinea pig colon (Costa et al., 2017), long distance contractions (LDCs) in the rat (Chen et al., 2013) and rabbit (Chen et al., 2016) or mass peristalsis in the rabbit colon (Lentle et al., 2008). The CMC can be evoked in the empty colon by an increase in intraluminal pressure in part or all of the colon. The CMC is a neurogenic motor pattern, yet the myogenic control system is an integral part of this complex (Bayguinov et al., 2010; Huizinga et al., 2011; Chen et al., 2013; Costa et al., 2013; Smith et al., 2014). The CMC involves the activation of interstitial cells of Cajal (Huizinga et al., 2011; Smith et al., 2014); Bayguinov et al. (2010) showed that it is dependent on a complex integration between myenteric neuronal and ICC-MP networks. The CMC is not dependent on slow wave activity from ICCDMP (Yoneda et al., 2002, 2004; Spencer et al., 2019),but it involves stimulus dependent pacemaker activity from ICC-MP (Bayguinov et al., 2010).

Peristalsis can also be evoked by a local luminal stimulus such as a pellet in the colon of a mouse or rabbit or aggregated contents in the human colon (Spencer et al., 2016). This is a reflex that can be called the Bayliss and Starling reflex (Bayliss and Starling, 1900; Spencer et al., 1999), and it was suggested that this reflex occurs because of the polarity of enteric neurons (Bornstein et al., 2004): pellets activate cholinergic excitatory neurons to stimulate contractions oral to the pellet, while simultaneously activating inhibitory nitrergic neurons anal to the pellet (Spencer et al.,
2002; Dickson et al., 2008). Nevertheless, it is not a simple reflex; bolus size and consistency affect propulsion speed, indicating that the features of the bolus-induced peristatic movement adapt to conditions (Costa et al., 2015). Although the neural component of the reflex is obvious, a role for a myogenic component, in particular related to the oral to anal direction of transit is likely (Huizinga et al., 2011; Sia et al., 2013). It was our objective to understand a potential role for butyrate in both of these mechanisms that can initiate peristalsis.

\section{MATERIALS AND METHODS}

\section{Animals}

All animal procedures were approved by the McMaster University Animal Research Ethics Board (AUP:14-12-49). 810-week-old female C57BL/6 mice were purchased from Jackson Laboratories. The animals were fed ad libitum and housed under standard conditions $\left(22 \pm 2^{\circ} \mathrm{C} ; 12 / 12\right.$-hr light/dark cycle) for at least 1 week prior to experiments.

\section{Colon Preparation}

C57BL/6 mice were euthanized by isofluorane inhalation followed by cervical dislocation. After opening the abdominal cavity, the whole colon was carefully excised and placed in oxygenated $\left(95 \% \mathrm{O}_{2}\right.$ and $\left.5 \% \mathrm{CO}_{2}\right)$ Krebs solution at $4^{\circ} \mathrm{C}$. The mesentery, blood vessels, and cecum were removed by ophthalmic scissors, and the fecal contents were flushed out by gavage with Krebs solution. Care was taken throughout these steps to minimize any stretching and disruptions to the mucosal layer.

\section{Colonic Motility and Pressure Recording}

The whole colon was transferred into the organ bath, which was filled with $600 \mathrm{ml}$ of oxygenated $\left(95 \% \mathrm{O}_{2}\right.$ and $\left.5 \% \mathrm{CO}_{2}\right)$ Krebs solution. The temperature of this Krebs solution was kept at $35^{\circ} \mathrm{C}$ through a heating tube which circulated water from an external water heater. The oral and anal ends were cannulated with an 18G needle and then fixed to the platform attached to the floor of the organ bath (Figure 1). The setup was also similar to a previously published photo (Parsons and Huizinga, 2015) and schematic drawing (Beck et al., 2019), for further reference. The oral end was connected to two plastic tubes, and the anal end was attached to a $1 \mathrm{ml}$ syringe without the plunger. At the anal end, each CMC caused outflow and was followed by minimal back flow. The pressure from fluid accunulating in the $1 \mathrm{~mL}$ syringe and its back flow was used to simulate endogenous conditions of increasing intraluminal pressure. The outflow height was allowed to reach a maximum of $3 \mathrm{~cm}$ above the anal end before any additional fluid was drained into a collection reservoir. At that point, fluid levels in the syringe fluctuated between 2.8 and $3 \mathrm{~cm}$ at most. At the oral end, one tube continuously perfused the colon with Krebs solution, while the other was connected to an intraluminal pressure transducer (Figure 1). This pressure port was located at the proximal-middle colonic junction (about $1 / 3$ from oral end) and the transducer was connected to a Grass LP 122 amplifier (Astro-Med, Brossard, 


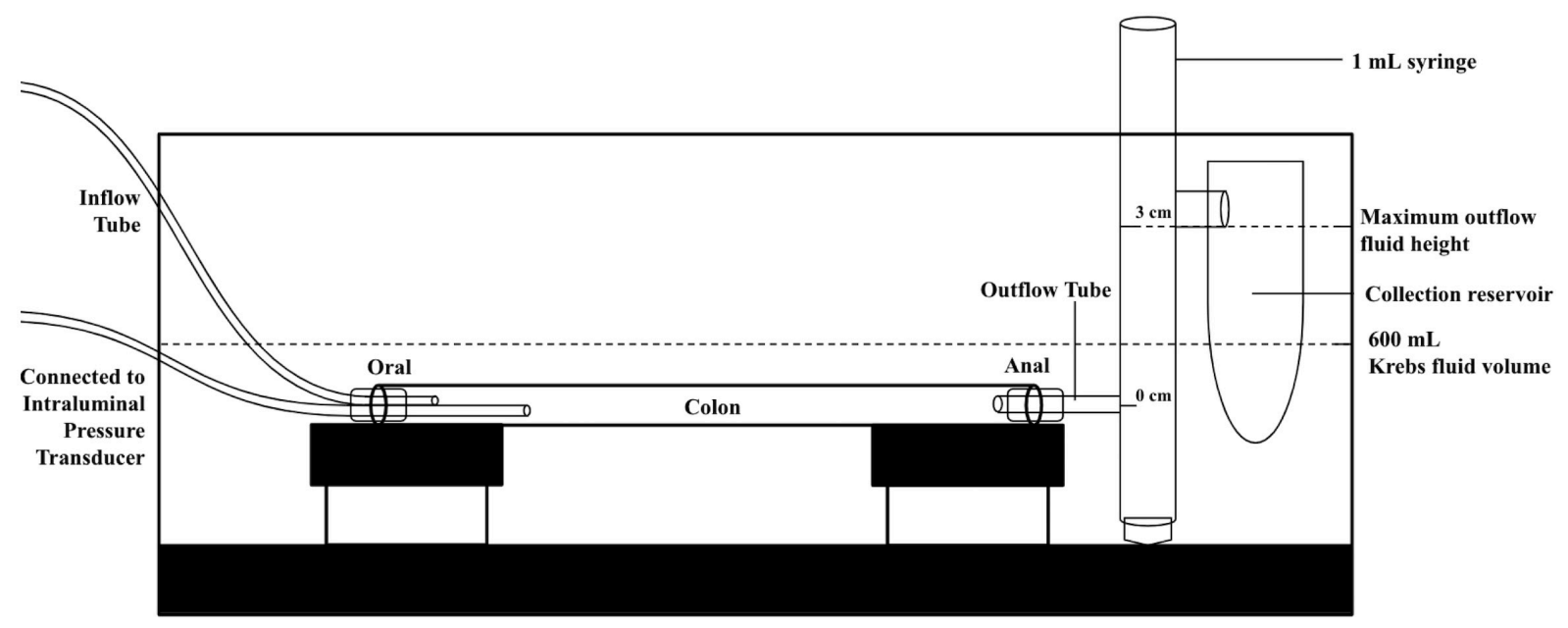

FIGURE 1 | Schematic of the colon bath setup to maintain $3 \mathrm{~cm}$ outflow pressure. For another experimental condition, the $1 \mathrm{~mL}$ syringe was replaced by an open collection reservoir to create $0 \mathrm{~cm}$ of outflow pressure.

QC, Canada). Its pressure signal was digitized using a MiniDigi 1A A-D converter, which was then displayed via Axoscope 10 software (Pclamp 10 software, Molecular Devices, Toronto, ON, Canada). The inflow perfusion was pumped into the lumen by a peristaltic pump (P-1, Pharmacia, Sweden) at an inflow rate of $30 \mu \mathrm{l} / \mathrm{min}$.

A miniature CDD camera (Effio CCD 700TVL, SONY, Hong Kong, China) was fixed above the preparation and used to record diameter changes indicative of colonic motility. The resulting videos were transferred to ImageJ for further analysis through a DMapLE plugin written by Dr. Sean Parsons. This software generated spatiotemporal maps of diameter changes (due to circular muscle contraction) over time. The diameter was assessed by measuring the distance between the two contrast borders at both sides of the colon (contrast between a white colon and black background). The colonic width was calculated at each point along the its length (image $Y$-axis), for each video frame (image $X$-axis) to generate the final DMap image. We used a gray scale with white as relaxation or distention and black as contraction (reduction in colon diameter).

\section{Intraluminal Solutions}

Krebs solutions contained (in $\mathrm{mM}$ ): $120 \mathrm{NaCl}, 6 \mathrm{KCl}, 15.5$ $\mathrm{NaHCO}_{3}, 1.2 \mathrm{NaH}_{2} \mathrm{PO}$, 0.1 citric acid, 0.1 aspartic acid,153 $2.5 \mathrm{CaCl}_{2}, 1.2 \mathrm{MgCl}_{2}$, and 6 glucose. A phosphate buffer solution (PBS) was used as an intraluminal inflow fluid, which contained (in mM): $117 \mathrm{NaCl}, 3.9 \mathrm{KCl}$, and $10 \mathrm{Na}_{2} \mathrm{HPO}_{4}$. Since glucose and high inflow rates can also induce motor activity through releasing 5-HT, PBS and low inflow rates provided the optimal conditions for viewing the effects of butyrate. $1 \mathrm{mM}, 10 \mathrm{mM}, 30 \mathrm{mM}$, and $100 \mathrm{mM}$ of sodium butyrate were dissolved into PBS solution, and Tris buffer was used to adjust $\mathrm{pH}$ values (7.35-7.45). Addition of 1$100 \mathrm{mM} \mathrm{NaCl}$ to PBS solution, did not change motor activity, consistent with a study by Hurst et al. (2014). Kendig et al.
(2014) also did not observe an effect of osmolarity on pellet propulsion in the rat.

\section{Experimental Design: General Distention and Butyrate Experiments}

After a 30-min acclimation period, colonic motor activity and intraluminal pressure changes were recorded. Two forms of general distention were used, by allowing the outflow tube to drain into an empty $1 \mathrm{ml}$ syringe placed vertically, its bottom flush with the colon. First, "increasing outflow pressure" was created by allowing all expelled Krebs solution to accumulate in the $1 \mathrm{~mL}$ outflow syringe. This fluid's rising height exerted increasing intraluminal pressure and distention over time. Second, we created "fixed outflow pressures" by maintaining the fluid height at $0 \mathrm{~cm}$ or $3 \mathrm{~cm}$ above the level of the colon.

For baseline, the outflow pressure was fixed at $0 \mathrm{cmH}_{2} \mathrm{O}$ and activity recorded for 15 to $20 \mathrm{~min}$. Interventions consisted of various solutions of butyrate and/or propionate added under both fixed and increasing outflow pressures. Activity under fixed outflow pressure was recorded for 15 to $20 \mathrm{~min}$, and activity for increasing outflow pressure was recorded for $40 \mathrm{~min}$ to allow for maximal fluid accumulation. The colon was given 10 min to acclimatize after each alteration in butyrate or propionate solutions.

\section{Experimental Design: Pellet-Induced Distention Experiments}

Artificial pellets of varying sizes (length: $10 \mathrm{~mm}$, diameter: 1.5$3 \mathrm{~mm}$ ) were made by silicone putty (EasyMold, Environmental Technology Inc., Fields County, CA, United States) and coated with black nail polish to ensure their visibility on the spatiotemporal maps. For each colon, the artificial pellet was size-matched with the endogenous pellets that were extracted during the cleaning process. Unlike with previous experiments, the colon was pinned directly to the floor of the organ bath, with 
its oral and anal ends kept open. After a 30-min acclimatization period, the baseline activity of the colon was recorded for $15 \mathrm{~min}$. A pellet was inserted into the oral end and its movements through the colon were recorded for $15 \mathrm{~min}$ or until it was expelled, upon which additional pellets would be inserted for a maximum of $30 \mathrm{~min}$. In other experiments, a polyethylene tube was inserted into the distal colon to allow perfusion with Krebs solution with or without butyrate for $15 \mathrm{~min}$. The pellet was then re-inserted under the aforementioned conditions and its movements recorded.

\section{Data Analysis and Statistical Methods}

All averaged values are presented as mean \pm SEM for parametric distributions, and median with interquartile range

TABLE 1 | The effect of increasing distention induced by outflow pressure on CMC frequency and diameter of the colon.

\begin{tabular}{|c|c|c|c|c|c|}
\hline & $0 \mathrm{~min}$ & $10 \mathrm{~min}$ & $20 \mathrm{~min}$ & $30 \mathrm{~min}$ & $40 \mathrm{~min}$ \\
\hline Frequency of CMCs (cpm) $(N=7)$ & 0 & $0.19 \pm 0.07$ & $0.33 \pm 0.07$ & $0.49 \pm 0.03$ & $0.44 \pm 0.04^{\mathrm{a}}$ \\
\hline Diameter $(\mathrm{mm})(N=7)$ & $1.66 \pm 0.06$ & $1.73 \pm 0.05$ & $1.92 \pm 0.06$ & $2.05 \pm 0.08$ & $2.21 \pm 0.11^{\mathrm{b}}$ \\
\hline CMC pressure amplitude $\left(\mathrm{cmH}_{2} \mathrm{O}\right)(\mathrm{N}=7)$ & 0 & $3.70 \pm 0.75$ & $5.94 \pm 1.19$ & $7.82 \pm 1.52$ & $8.33 \pm 1.14^{\mathrm{C}}$ \\
\hline Outflow pressure $\left(\mathrm{cmH}_{2} \mathrm{O}\right)(\mathrm{N}=7)$ & 0 & $1.44 \pm 0.13$ & $3.46 \pm 0.20$ & $5.37 \pm 0.31$ & $6.86 \pm 0.24^{d}$ \\
\hline
\end{tabular}

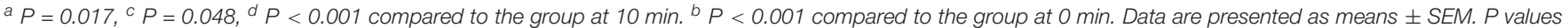
were determined by a one-way ANOVA and Tukey's post hoc test.

TABLE 2 | Velocities of motor patterns with and without Krebs perfusion.

\begin{tabular}{|c|c|c|c|c|c|}
\hline Group & $\begin{array}{c}\text { Number of pellets } \\
\text { inserted }\end{array}$ & $\begin{array}{l}\text { Pellet expulsion } \\
\text { (number) }\end{array}$ & $\begin{array}{c}\text { Velocity of pellet } \\
\text { movements }(\mathrm{mm} / \mathrm{s})\end{array}$ & $\begin{array}{c}\text { Velocity of CMCs } \\
(\mathrm{mm} / \mathrm{s})\end{array}$ & $\begin{array}{c}\text { Velocity of reflex } \\
\text { movements }(\mathrm{mm} / \mathrm{s})\end{array}$ \\
\hline \multirow[t]{4}{*}{ No Krebs $(N=7)$} & 20 & Yes (13) & $0.32 \pm 0.03^{a}$ & $0.34 \pm 0.03$ & $0.20 \pm 0.04$ \\
\hline & & & $(n=56)$ & $(n=47)$ & $(n=9)$ \\
\hline & & No (7) & $0.18 \pm 0.01$ & $0.21 \pm 0.01$ & $0.06 \pm 0.01$ \\
\hline & & & $(n=37)$ & $(n=30)$ & $(n=7)$ \\
\hline \multirow{4}{*}{$\begin{array}{l}\text { Krebs } \\
(N=17)\end{array}$} & 41 & Yes (27) & $0.39 \pm 0.03$ & $0.41 \pm 0.03$ & $0.37 \pm 0.04$ \\
\hline & & & $(n=76)$ & $(n=26)$ & $(n=50)$ \\
\hline & & No (14) & $0.29 \pm 0.02$ & $0.35 \pm 0.03$ & $0.23 \pm 0.02$ \\
\hline & & & $(n=34)$ & $(n=16)$ & $(n=18)$ \\
\hline
\end{tabular}

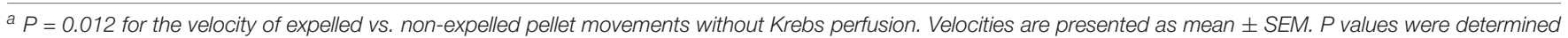
by a one-way ANOVA and Tukey's post hoc test.

TABLE 3 | Frequencies of motor patterns with and without Krebs perfusion.

\begin{tabular}{|c|c|c|c|c|c|}
\hline Group & $\begin{array}{c}\text { Number of } \\
\text { pellets }\end{array}$ & $\begin{array}{l}\text { Expulsion } \\
\text { Time (min) }\end{array}$ & $\begin{array}{c}\text { Number of pellet } \\
\text { movements }\end{array}$ & $\begin{array}{l}\text { Frequency of CMCs } \\
\text { (cpm) }\end{array}$ & $\begin{array}{l}\text { Frequency of reflex } \\
\text { movements (cpm) }\end{array}$ \\
\hline No Krebs $(N=7)$ & 13 & $15.4 \pm 2.2(n=13)$ & $4.3 \pm 0.5(n=13)$ & $0.21(0.18,0.29)(n=13)$ & $0.06(0.05,0.11)(n=7)$ \\
\hline Krebs $(N=17)$ & 27 & $7.5 \pm 0.9^{\mathrm{a}}(n=27)$ & $2.8 \pm 0.2^{\mathrm{b}}(n=27)$ & $0.16(0.12,0.23)(n=14)$ & $0.25(0.14,0.68)^{c}(n=23)$ \\
\hline
\end{tabular}

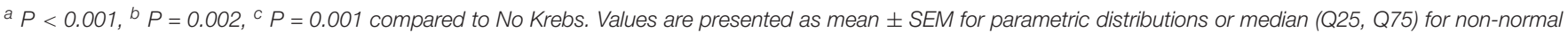

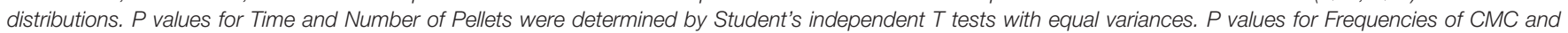
Reflex Movements were determined by Wilcoxon-Mann-Whitney tests.

TABLE 4 | Velocities of motor patterns in the control and butyrate groups.

\begin{tabular}{|c|c|c|c|c|c|}
\hline Group & $\begin{array}{c}\text { Number of } \\
\text { pellets inserted }\end{array}$ & $\begin{array}{l}\text { Pellet expulsion } \\
\text { (number) }\end{array}$ & $\begin{array}{c}\text { Velocity of pellet } \\
\text { movements }(\mathrm{mm} / \mathrm{s})\end{array}$ & $\begin{array}{c}\text { Velocity of CMCs } \\
(\mathrm{mm} / \mathrm{s})\end{array}$ & $\begin{array}{c}\text { Velocity of reflex } \\
\text { movements }(\mathrm{mm} / \mathrm{s})\end{array}$ \\
\hline \multirow[t]{2}{*}{ Control $(N=10)$} & 21 & Yes (16) & $\begin{array}{c}0.34 \pm 0.03 \\
(n=49)\end{array}$ & $\begin{array}{c}0.41 \pm 0.38 \\
(n=17)\end{array}$ & $\begin{array}{c}0.30 \pm 0.04 \\
(n=32)\end{array}$ \\
\hline & & No (5) & $\begin{array}{c}0.28 \pm 0.04 \\
(n=9)\end{array}$ & $\begin{array}{c}0.45 \pm 0.05 \\
(n=2)\end{array}$ & $\begin{array}{c}0.23 \pm 0.05 \\
(n=7)\end{array}$ \\
\hline \multirow[t]{2}{*}{ Butyrate $(N=10)$} & 23 & Yes (21) & $\begin{array}{c}0.39 \pm 0.03 \\
(n=56)\end{array}$ & $\begin{array}{c}0.42 \pm 0.05 \\
(n=20)\end{array}$ & $\begin{array}{c}0.38 \pm 0.04 \\
(n=36)\end{array}$ \\
\hline & & No (2) & $\begin{array}{c}0.34 \pm 0.02 \\
(n=3)\end{array}$ & - & $\begin{array}{c}0.34 \pm 0.02 \\
(n=3)\end{array}$ \\
\hline
\end{tabular}

$P$ values (NS) were determined by a one-way ANOVA and Tukey's post hoc test. Values are presented as mean \pm SEM. 
TABLE 5 | Frequencies of motor patterns in the control and butyrate groups.

\begin{tabular}{|c|c|c|c|c|c|}
\hline Group & $\begin{array}{c}\text { Number of pellets } \\
\text { inserted }\end{array}$ & $\begin{array}{l}\text { Expulsion Time } \\
\text { (min) }\end{array}$ & $\begin{array}{c}\text { Number of pellet } \\
\text { movements }\end{array}$ & $\begin{array}{l}\text { Frequency of CMCs } \\
\text { (cpm) }\end{array}$ & $\begin{array}{l}\text { Frequency of reflex } \\
\text { movements (cpm) }\end{array}$ \\
\hline Control $(N=10)$ & 16 & $8.4 \pm 1.0(n=16)$ & $3.1 \pm 0.3(n=16)$ & $0.14(0.12,0.19)(n=10)$ & $0.19(0.12,0.40)(n=15)$ \\
\hline Butyrate $(N=10)$ & 21 & $5.6 \pm 0.9^{a}(n=21)$ & $2.7 \pm 0.3(n=21)$ & $0.32(0.24,0.43)(n=4)$ & $0.46(0.30,0.66)^{\mathrm{b}}(n=18)$ \\
\hline
\end{tabular}

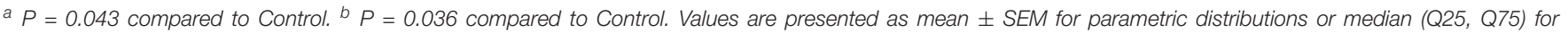

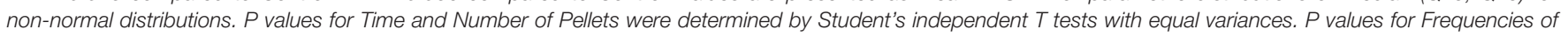
CMC and Reflex Movements were determined.

(Q25, Q75) for non-parametric distributions. Statistical tests for significance were performed with SPSS (Chicago, Ill. United States) and Real Statistics (Charles Zaiontz). In Tables 1, 2, and 4, one-way ANOVA with Tukey's post hoc tests were used to derive specific $P$ values. For Tables 3 and 5, the differences in expulsion time and the number of pellet movements were compared using Student's Independent $T$ tests, with equal variances. The differences between the frequencies of CMCs and frequencies of reflexes in the pellet experiments (Tables 3 and 5) were compared by WilcoxonMann-Whitney tests, using exact $P$ values. To compare the effects of butyrate at $0 \mathrm{~cm}$ vs. $3 \mathrm{~cm}$ of fixed distention, Student's Independent $T$ tests with equal variances were used. In the sudden distention experiments, the Student's Paired $T$ test was used to compare the same sample before and after an intervention.

One-way ANOVA with Tukey's post hoc tests were used for all $P$ value calculations involving propionate, except for the frequency of CMCs at the baseline and after $30 \mathrm{mM}$ of propionate, where $P$ values were calculated by Student's Independent $T$ tests assuming equal variances. A $P$ value of 0.05 is considered to indicate significance. Figures were created with GraphPad PRISM software (La Jolla, CA, United States).

\section{Calculations}

The diameter of the colon at any time was taken from the $z$ axis of its spatiotemporal map. CMCs and reflexes were analyzed in terms of their amplitudes, frequencies and velocities. The amplitudes of CMCs and reflexes were calculated by measuring the maximum change in diameter at a fixed point along the length of the colon during each motor pattern. CMC frequencies and reflexes were calculated by dividing the number of motor patterns by the minutes over which they occurred. The velocities were calculated by taking the slope of a diagonal line drawn from the start to end of a spatiotemporal map of the motor pattern, located on a $y$ axis indicating distance and an $x$ axis indicating time.

Additionally, measures of pressure were also taken, including the outflow pressure, basic intraluminal pressure and pressure amplitude. The outflow pressure was recorded by examining the fluid level in the outflow syringe which had accumulated above the level of the colon $\left(0 \mathrm{cmH}_{2} \mathrm{O}\right)$. The basic intraluminal pressure was the lowest intraluminal pressure reading in each recorded session. Intraluminal pressure amplitude was calculated as the difference between the maximum and minimum intraluminal pressure values during any given motor pattern.

\section{Nomenclature}

The nomenclature used for colonic propulsive motor patterns in animal models is not consistent in the literature. A major propulsive motor pattern is a pan-colonic rhythmic activity that has been called, amongst others, "colonic migrating motor complex," "mass peristalsis" or "giant contractions" occurring at a frequency around $0.5 \mathrm{cpm}$ in the mouse (Powell et al., 2003) and rabbit colon (Quan et al., 2017) and up to $2 \mathrm{cpm}$ in the guinea pig (Costa et al., 2015). At a consensus meeting it was agreed to use the term "CMC" (Corsetti et al., 2019). We recently demonstrated in the rabbit colon that the characteristics of this motor complex are dramatically different at different levels of excitation (Shokrollahi et al., 2019). In the rabbit, mouse and rat, we and others have identified the long distance contraction (LDC) as the most forceful circumferential propulsive contraction evoked by distention that propels content down the colon; it is the most forceful expression of the CMC (Chen et al., 2013; Kendig and Grider, 2015; Yu et al., 2015; Li et al., 2016; Vincent et al., 2018). The propulsive nature of the LDC can be measured by its association with 3-5 ml outflow (Chen et al., 2016). LDCs have a characteristic triangular shape in spatiotemporal maps, very similar in the rabbit, mouse and rat, due to the fact that the most proximal part of the colon remains contracted while the front of the contraction propagates anally, preceded by relaxation (Chen et al., 2013). LDCs are neurogenic in that they are abolished by TTX or hexamethonium (Lentle et al., 2008; Chen et al., 2016). The second most prominent propulsive activity, observed under less excited conditions is the fast propagating contraction (FPC), often occurring in clusters. FPCs are circular muscle contractions that occur at a higher frequency and propagate at a higher velocity compared to LDCs and propagate usually in antegrade direction. They are less forceful than LDCs and they may occur in clusters of several FPCs (Quan et al., 2017). FPCs are also called "fast phasic activity" (Lentle et al., 2008) and clusters of FPCs have been associated with 1-3 ml outflow per FPC (Chen et al., 2016). In the rabbit colon it has been determined that single FPCs are myogenic (Lentle et al., 2008), but the forming of FPC clusters is neurogenic (Chen et al., 2016). LDCs and FPCs are two manifestations of CMCs that will be described in this paper.

In addition to these two motor patterns, there are three other motor patterns in the mouse colon, previously characterized in the rat colon: myogenic ripples (Chen et al., 2013), proximal rhythmic contractions (Li et al., 2016) and distal rhythmic propagating motor complexes (Chen et al., 2013; Li et al., 2016). 
A

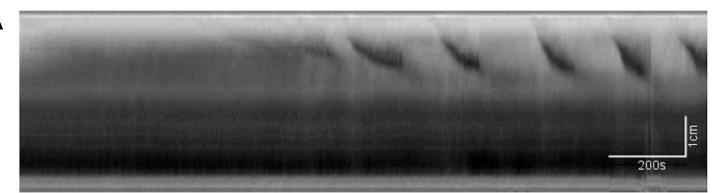

B

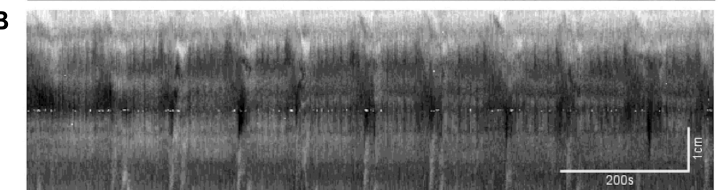

C

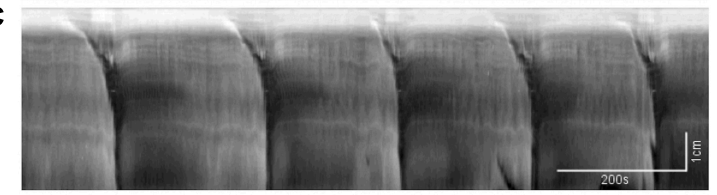

D

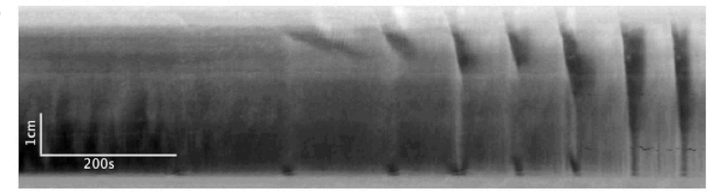

E

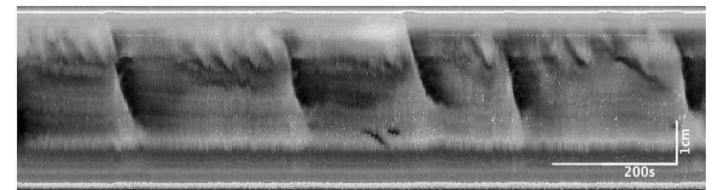

$\mathbf{F}$

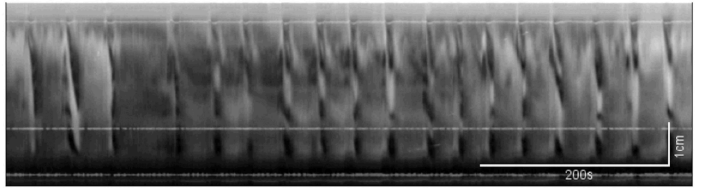

FIGURE 2 | The colonic motor complex (CMC) at different levels of excitation. In this and all subsequent figures, black identifies contraction, and white relaxation or distention; the top of all figures is the proximal part of the colon. (A) Upon mild excitation by increased intraluminal pressure, proximal rhythmic (pacemaker) activity appear in the colon always starting in the most proximal pacemaker activity. These are usually rhythmic, and almost always start in the proximal colon to propagate a short distance. (B) Fast propagating contractions (FPCs). Further excitation may elicit a CMC, which is always rhythmic and triggered by proximal activity. The weakest form of CMCs presented as single FPCs, clustered-FPCs or a mix of short contractions that together form a complex of pan-colonic activity. (C) Long distance contractions (LDCs). Full-blown LDCs are shown here, which are manifestations of CMCs at their highest level of excitation. (D) This figure shows how proximal rhythmic contractions can develop from proximal activity to eventually trigger LDCs. (E) This figure shows how proximal activity can occur rhythmically and at a high frequency where only every 3rd or 5th activity triggers an LDC. (F) "Broken" long distance contractions. The CMC can show many compositions in between low-amplitude FPCs and full-blown LDCs. Here we see many LDCs we have previously identified as "broken LDCs" (Chen et al., 2013), where it appears that the LDC is interrupted by one or many streaks of inhibition.

\section{RESULTS}

\section{The Motor Patterns of the Mouse Colon at Different Levels of Excitation}

Figure 2 shows examples of the CMC at different levels of excitation. These motor complexes are all pan-colonic and occur in a rhythmic manner. Without any stimulation, the mouse colon often exhibited rhythmic proximal activity (Figure 2A). Colons that were placed in the organ bath without any stretch or distention showed CMC activity in all preparations (Figure 2B). The weakest form of CMCs presented as single FPCs, clusteredFPCs or a mix of short contractions that together formed a complex of pan-colonic activity. Upon further distention, these weaker motor patterns then changed into various configurations with increasing distention, up to its most excited configuration, the LDC (Figures 2A-F).

\section{Distention-Induced Changes in the Colonic Motor Complex}

Distention-mediated excitation was accomplished in three different ways.

The Colon Was Perfused With Krebs Solution and the Outflow Collected in a Vertical Tube so That the Intraluminal Pressure Gradually Increased.

In this series of experiments, the outflow pressure increased gradually from 0 to $\sim 7 \mathrm{cmH}_{2} \mathrm{O}(\mathrm{N}=7)$ (Figure 3 and Table $\mathbf{1}$ ). The resultant change in mean intraluminal pressure did not increase to the same degree: the mean intraluminal pressure started at $1.47 \pm 0.43$ and increased to $4.16 \pm 0.59 \mathrm{cmH}_{2} \mathrm{O}$, likely due to adaptive relaxation in the colon $(N=7)$. With increasing intraluminal pressure, the CMCs progressed from weak simultaneous contractions to FPCs, culminating in LDCs (Figures 2C,D, 3). The CMC pressure amplitude increased from $3.70 \pm 0.75$ to $8.33 \pm 1.14 \mathrm{cmH}_{2} \mathrm{O}(p=0.048, N=7, n=28)$, accompanied by a significant increase in the average frequency of CMCs from $0.19 \pm 0.07$ to $0.44 \pm 0.04 \mathrm{cpm}(p=0.017, N=7$, $n=28)$ (Table 1).

\section{The Colon Was Perfused With Krebs Solution With the Outflow Fixed at $0 \mathrm{cmH}_{2} \mathrm{O}$ Above the Colon}

With outflow levels fixed at $0 \mathrm{cmH}_{2} \mathrm{O}$, the basic intraluminal pressure was $1.94 \pm 0.44 \mathrm{cmH}_{2} \mathrm{O}(N=9, n=7)$ due to a constant infusion of Krebs solution, and the average colonic diameter was $1.73 \pm 0.09 \mathrm{~mm}(N=9, n=9)$ (Figure 4). Proximal rhythmic contractions and FPCs were the predominant motor patterns observed during this phase (Figure 5A), with LDCs present in 2 experiments.

Under these conditions, $1 \mathrm{mM}$ butyrate increased the frequency of CMCs from $0.16 \pm 0.04$ to $0.51 \pm 0.03 \mathrm{cpm}$ $(p<0.001, N=10, n=42)$, but no significant increases in amplitude were observed (Figures 5A,C). $10 \mathrm{mM}$ of butyrate also increased the frequency of CMCs from $0.16 \pm 0.04$ to $0.41 \pm 0.03 \mathrm{cpm}(p<0.001, N=10, n=42)$ No significant changes in CMC frequency nor amplitude were found with additional butyrate by comparison (30 mM, 100 mM, Figure 5C).

\section{The Colon Was Perfused With Krebs Solution With the Outflow Fixed at $3 \mathrm{cmH}_{2} \mathrm{O}$ Above the Colon} When the outflow level was fixed at $3 \mathrm{cmH}_{2} \mathrm{O}$, the basic intraluminal pressure was $2.47 \pm 0.90 \mathrm{cmH}_{2} \mathrm{O}(N=7, n=5)$ with a diameter of $1.94 \pm 0.04 \mathrm{~mm}(N=7, n=7$, Figure 4). Under these conditions, all experiments exhibited pan-colonic 


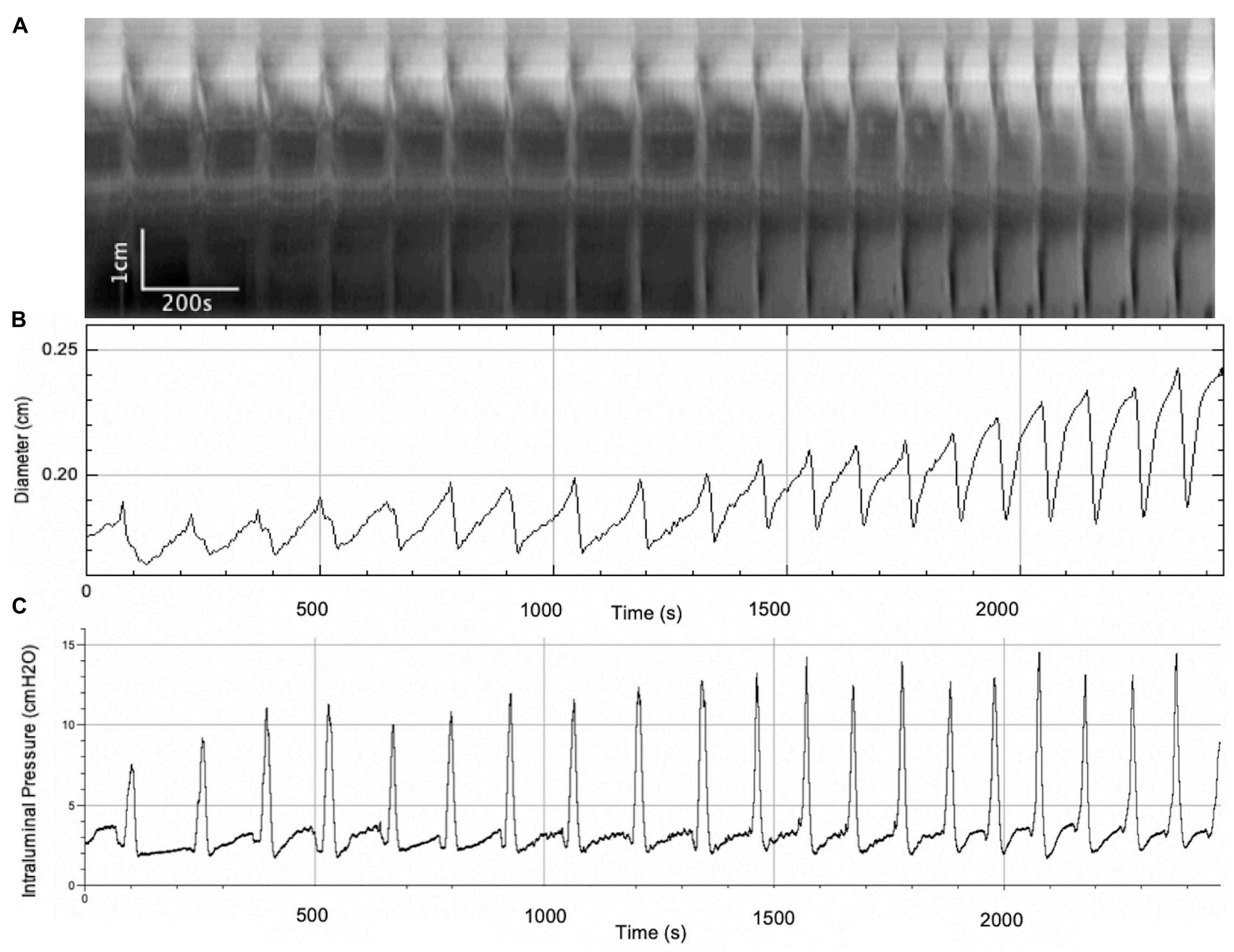

FIGURE 3 | A gradual increase in intraluminal pressure causes an increase in CMC frequency and the amplitude of accompanying phasic contractions, as well as intraluminal pressure changes. (A) Spatiotemporal map of CMCs. (B) Average diameter of the colon over time. (C) Intraluminal pressure in the colon over time.

motor complexes in the form of either FPCs or LDCs (Figure 5B). There was also a $2.42 \pm 0.86 \mathrm{cmH}_{2} \mathrm{O}$ increase $(p=0.019, N=13$, $n=13$ ) in the pressure amplitude of CMCs and a $0.22 \pm 0.06 \mathrm{cpm}$ increase in the average frequency $(p=0.002, N=17, n=17)$ of pan-colonic CMCs in comparison to mice under $0 \mathrm{cmH}_{2} \mathrm{O}$ of outflow pressure (Figures 4A,B).

Butyrate at $1-100 \mathrm{mM}$ was administred to these preparation $(N=19)$ but none were capable of significantly increasing the frequency of CMCs observed (Figure 5B). Additionally, changes in CMC frequency and amplitude were not consistently correlated to increasing concentrations of butyrate during high distention (Figure 5C).

\section{Transient Local Distention Occurring Due to a Sudden Blockage of Outflow}

On three occasions, sudden local distention occurred in the middle of the colon due to a blockage of outflow by gas bubbles or dislodged mucosa (Figure 6A). The average diameter of the colon increased from $1.83 \pm 0.29$ to $2.73 \pm 0.41 \mathrm{~mm}(N=3, n=6, p=0.054)$ (Figure 6B). This local distention augmented colonic motor activity by generating (a) regular LDCs developing from proximal contractions or (b) strong LDCs from weak, pre-existing LDCs (Figure 6A). The frequencies of LDCs were significantly increased from $0.23 \pm 0.04$ to $0.60 \pm 0.11 \mathrm{cpm}(N=3$, $n=6, p=0.035)$. When these blockages were spontaneously resolved, the previous weaker LDCs and proximal contractions returned.

\section{Butyrate-Induced Changes in the Colonic Motor Complex in the Presence of Propionate and Distention}

Butyrate in the Presence of Propionate at $0 \mathrm{cmH}_{2} \mathrm{O}$ of Outflow Pressure

In these experiments, the outflow pressure of the colon was maintained at $0 \mathrm{cmH}_{2} \mathrm{O}$, giving an average intraluminal pressure of $1.36 \pm 0.20 \mathrm{cmH}_{2} \mathrm{O}(\mathrm{N}=9)$. In this low-distention state, $30 \mathrm{mM}$ of propionate decreased the average frequency of CMCs by $0.12 \pm 0.05 \mathrm{cpm}(p=0.031, N=9, n=18)$. CMCs initially present were either completely abolished or reduced to proximal rhythmic contractions (Figure 7). Accordingly, their frequencies were either reduced to $0 \mathrm{cpm}(N=5)$, decreased by $0.13 \pm 0.03 \mathrm{cpm}(N=2)$ or increased by 


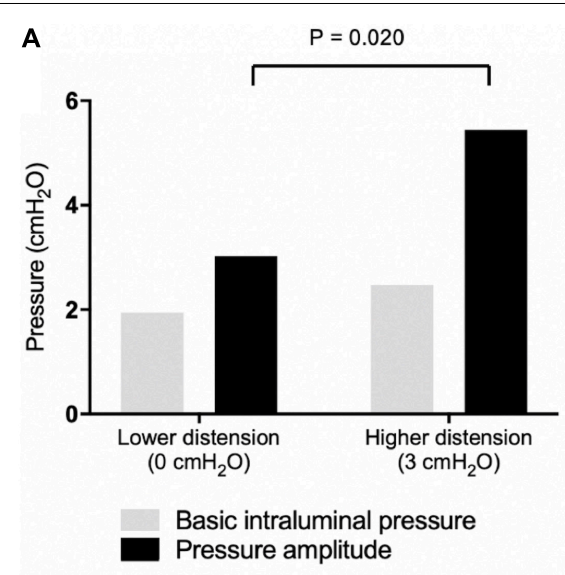

B

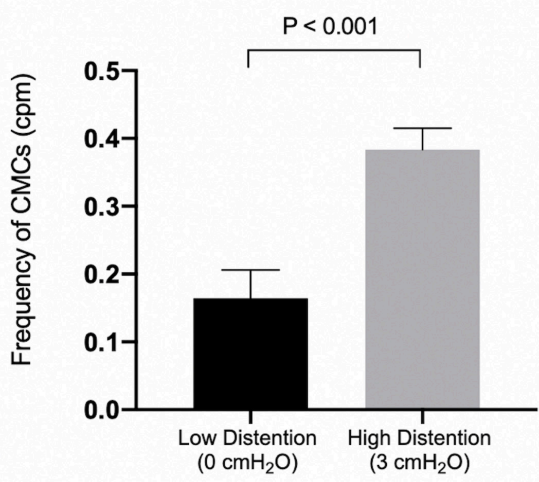

C

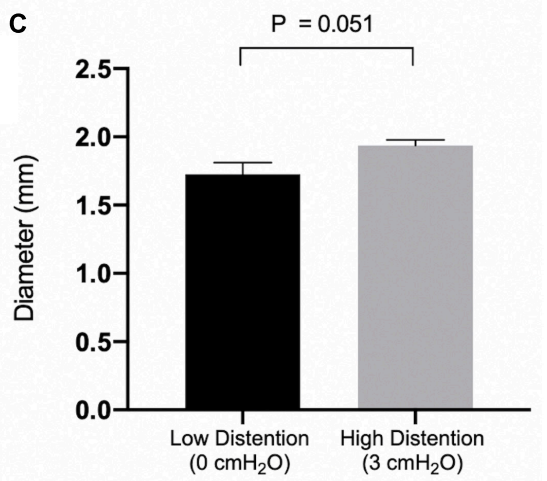

FIGURE 4 | (A) Basic intraluminal pressure and pressure amplitudes of observed $\mathrm{CMCs}$ under low and high distention. $\mathrm{Compared} \mathrm{with} 0 \mathrm{cmH} \mathrm{H}_{2} \mathrm{O}(\mathrm{N}=7)$, $3 \mathrm{cmH} \mathrm{H}_{2} \mathrm{O}$ $(N=5)$ of outflow pressure increased the pressure amplitude of $\mathrm{CMCs}$ observed $(P=0.020)$. (B) $\mathrm{CMC}$ frequencies under low and high distention. Compared with $\mathrm{O} \mathrm{cmH}_{2} \mathrm{O}(\mathrm{N}=10), 3 \mathrm{cmH}_{2} \mathrm{O}(N=7)$ of outflow pressure increased the frequency of $\mathrm{CMCs}$ in the colon $(P<0.001$. (C) Colonic diameter under low $(N=9)$ and high distention $(N=7)$. $P$ values were determined by Student's $T$ test.

$0.08 \pm 0.06 \mathrm{cpm}$ with lowered amplitudes (diameter reduction) by $1.55 \pm 0.21 \mathrm{~mm}(N=2)$.

$30 \mathrm{mM}$ butyrate was added after $30 \mathrm{mM}$ propionate perfused the colon in a low distention state, with an average intraluminal pressure of $2.79 \pm 0.46 \mathrm{cmH}_{2} \mathrm{O}$ induced by maintaining 0 $\mathrm{cmH}_{2} \mathrm{O}$ of outflow pressure $(N=5)$. Increases in CMC frequency of $0.40 \pm 0.16 \mathrm{cpm}$ were seen in 3 mice $(N=3)$. Overall, adding $30 \mathrm{mM}$ butyrate to $30 \mathrm{mM}$ propionate increased the amplitude of CMCs by $0.04 \pm 0.01 \mathrm{~cm}(p=0.009, N=5, n=32$, Figure 8B). However, it had inconsistent effects on CMC frequency at a low distention state (Figure 8A).

\section{Butyrate in the Presence of Propionate Under Increasing Distention Induced by Outflow Pressure}

In these experiments, the outflow pressure was allowed to accumulate from 0 to 3 or $5 \mathrm{~cm}$. Motor patterns at baseline began as weak myogenic ripples or proximal rhythmic contractions. Increased distention induced strong, high amplitude proximal rhythmic contractions, which then progressed into weak CMCs in the form of FPCs, and finally LDCs at the highest level of distention (Figures 8, 9). CMC frequencies increased by $0.23 \pm 0.07 \mathrm{cpm}(p=0.007, N=6$, $n=12$ ) after $10 \mathrm{~min}$ and $0.36 \pm 0.07 \mathrm{cpm}$ after $20 \mathrm{~min}$ $(p<0.001, N=6, n=12)$ (Figure 10A). The amplitudes of CMCs increased by $0.058 \pm 0.015 \mathrm{~cm}(p=0.002, N=6$, $n=28)$ upon adding $30 \mathrm{mM}$ propionate and decreased by $0.046 \pm 0.015 \mathrm{~cm}(p=0.020, N=6, n=24)$ after $20 \mathrm{~min}$ (Figure 10B).

$30 \mathrm{mM}$ butyrate was added with $30 \mathrm{mM}$ propionate under increasing outflow pressure ( 0 to $\sim 3$ or $5 \mathrm{cmH}_{2} \mathrm{O}$ ), as described above $(N=8)$. Compared to propionate alone, butyrate coupled with distention was able to significantly increase CMC frequencies $0.21 \pm 0.07 \mathrm{cpm}(p=0.045, N=8, n=16)$ after $20 \mathrm{~min}$ (Figure 11). Butyrate surpassed baseline CMC frequencies by $0.30 \pm 0.07 \mathrm{cpm}(p=0.002, N=8, n=16)$ after $30 \mathrm{~min}$ (Figure 11). However, these changes were not significantly different from those induced solely by distention in the presence of propionate.

\section{Motor Patterns Associated With Pellet Expulsion and Butyrate}

Pellet movements occurred through two distinctly different motor activities. Most pellet movements occurred through CMCs. Almost always, a CMC would move the pellet for a short distance and then the CMC would continue without the 

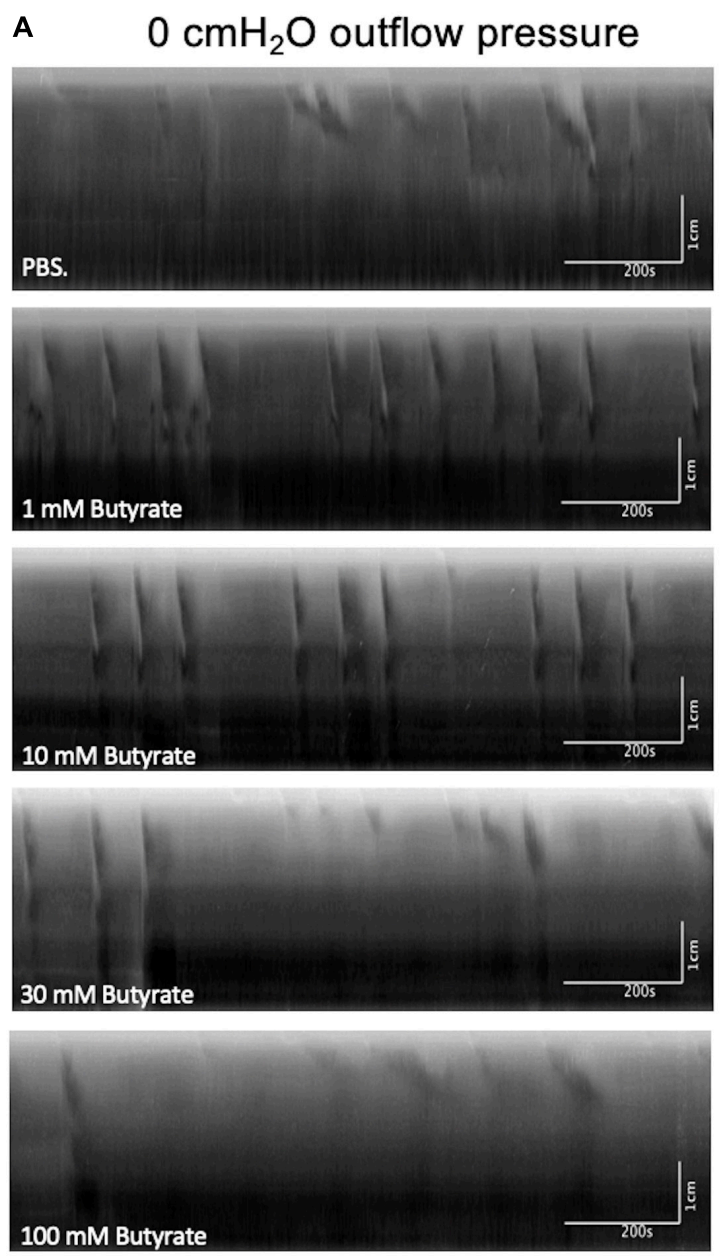

C

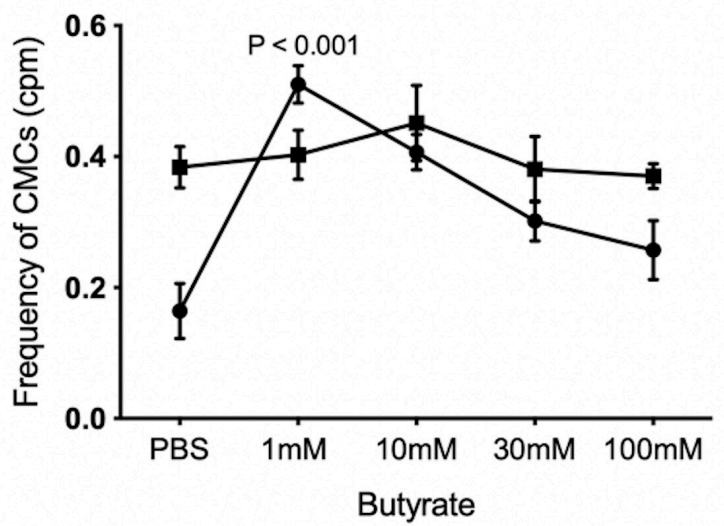

B $3 \mathrm{cmH}_{2} \mathrm{O}$ outflow pressure
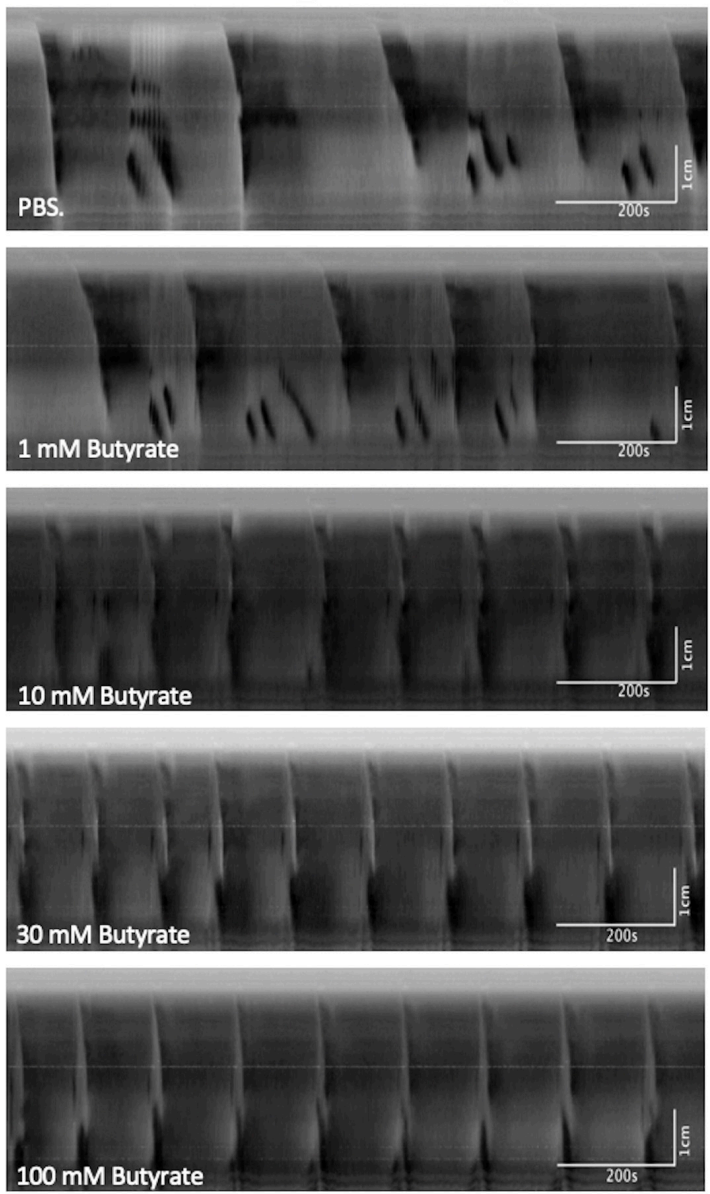

$\rightarrow$ Low distension $\left(0 \mathrm{cmH}_{2} \mathrm{O}\right)$

$\rightarrow$ High distension $\left(3 \mathrm{cmH}_{2} \mathrm{O}\right)$

FIGURE 5 | The effect of butyrate at fixed levels of high and low distention on CMCs. (A) Spatiotemporal maps showing the effects of increasing butyrate concentrations on colonic motor activity under low distention. Administering $1 \mathrm{mM}$ of luminal butyrate caused proximal rhythmic contractions to extend into pan-colonic CMCs, specifically FPCs. LDCs were formed at $10 \mathrm{mM}$ of butyrate but disappeared upon continued increases in butyrate concentration to $30 \mathrm{mM}$ or $100 \mathrm{mM}$. (B) Spatiotemporal maps showing the effects of increasing butyrate concentrations on colonic motor activity under high distention. Administering $10 \mathrm{mM}$ of butyrate increased CMC frequency on average. Further increases in CMC frequencies were inconsistent in response to 30-100 mM butyrate. (C) Frequency of CMCs in response to varying concentrations of butyrate in high and low distention. Butyrate $(1 \mathrm{mM})$ significantly increases $\mathrm{CMC}$ frequency in instances of low intraluminal pressure and activity, as simulated by low distention $\left(0 \mathrm{cmH}_{2} \mathrm{O}\right.$ of outflow pressure $(\mathrm{N}=10, P<0.001)$. Increasing the concentration of butyrate to $10 \mathrm{mM}, 30 \mathrm{mM}$ or $100 \mathrm{mM}$ did not further augment CMC frequency. Butyrate has a negligible effect on CMC frequency in instances of high intraluminal pressure and baseline motor activity, as simulated by high distention $\left(3 \mathrm{cmH}_{2} \mathrm{O}\right.$ outflow pressure) $(N=7)$. $P$ values were determined by a one-way ANOVA and Tukey's post hoc test. 


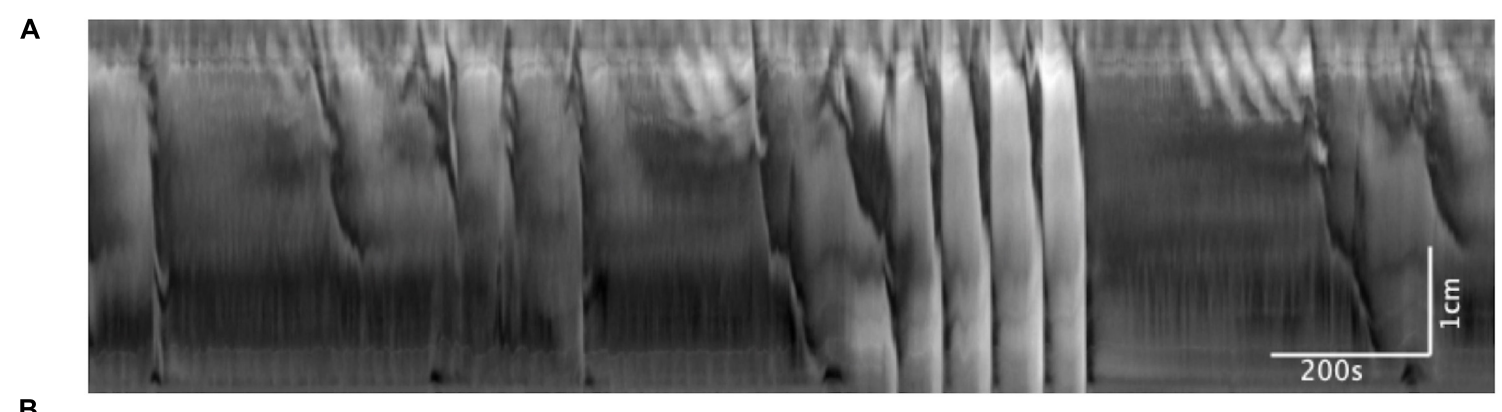

B

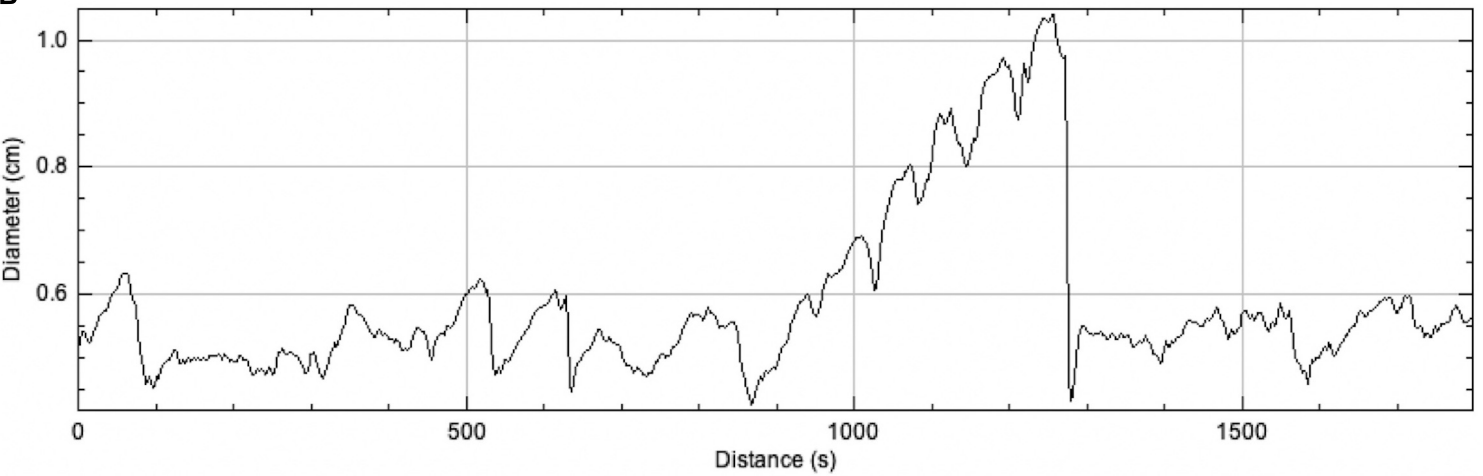

FIGURE 6 | The effect of sudden distention on colonic motor activity caused by a blockage in outflow. When this occurred, LDCs immediately developed to expel the blockage and restore baseline activity levels. (A) Spatiotemporal map. (B) Average colonic diameter over time.

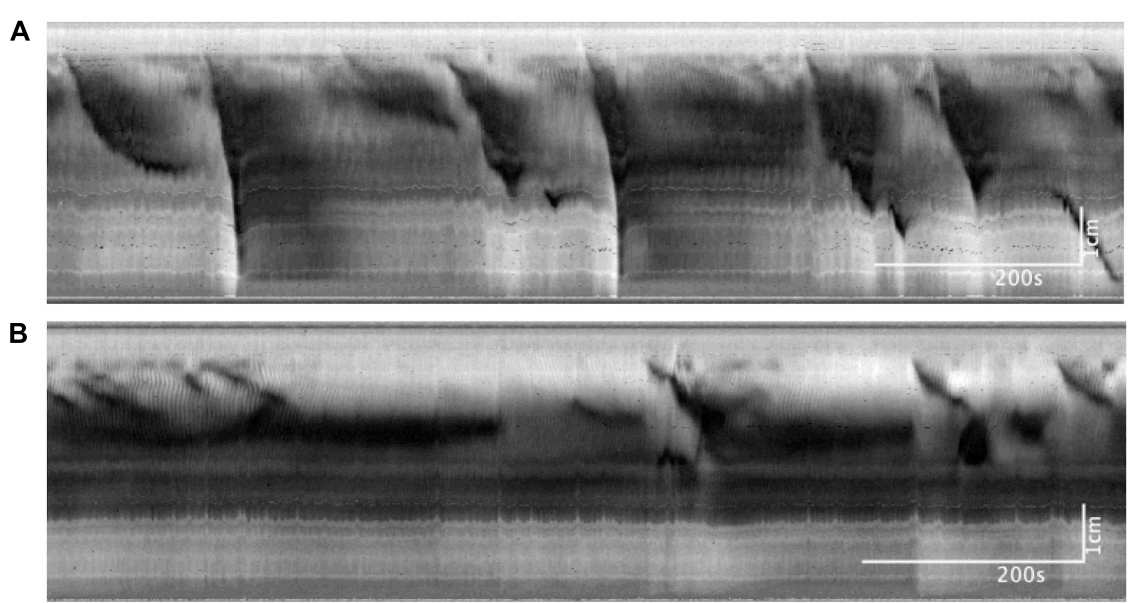

FIGURE 7 | Colonic motor activity at baseline compared to $30 \mathrm{mM}$ of luminally administered propionate. (A) Baseline. (B) Propionate (30 mM). Compared to baseline conditions, $30 \mathrm{mM}$ propionate decreases LDCs and FPCs, while leaving proximal rhythmic activity intact.

pellet. In addition, a second type of motor activity was induced by the pellet, termed the reflex movement, the classical Bayliss and Starling peristaltic reflex; a ring contraction developed orally to the pellet (Figure 12A), which was seen in the spatiotemporal maps as a black band (Figure 12B), which then moved the pellet (Bayliss and Starling, 1900). The pellet would stop moving once the ring contraction subsided. The ring contraction was often preceded by a relaxation, which can be observed in the spatiotemporal maps as a short retrograde movement of the pellet (Figure 12B). Rarely was the pellet moved out of the colon by a single CMC or a single reflex movement.

\section{Pellet Movement in a Non-distended Colon Without Krebs Perfusion}

In seven mice, 20 pellets moved out of the colon within $15.40 \pm 2.23 \mathrm{~min}$, through $47 \mathrm{CMCs}$ and nine reflex movements (Figure 13). When the pellet moved along the colon but did not expel within $30 \mathrm{~min}$, the experiment was terminated; this occurred with seven pellets that moved through only $61 \pm 2 \%$ 


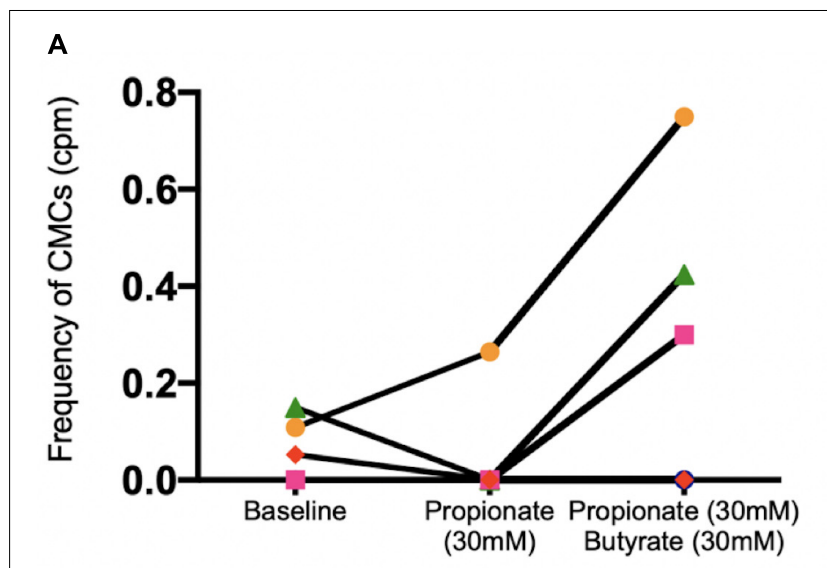

B

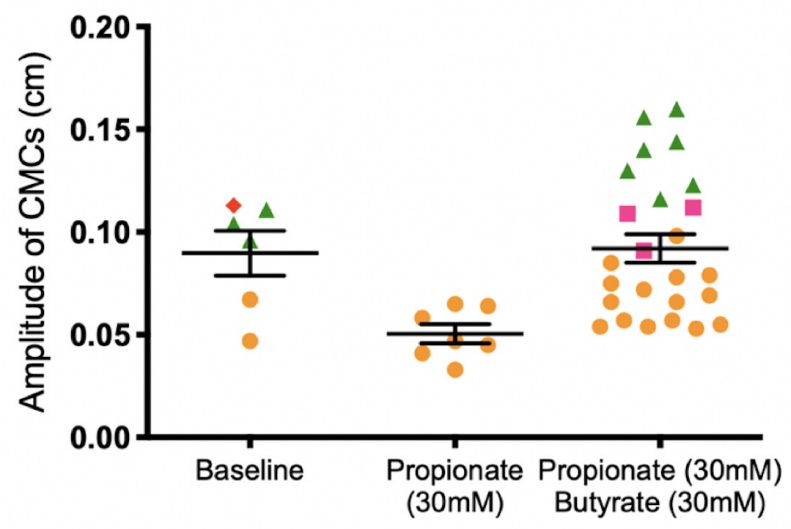

FIGURE 8 | Colonic motor activity before and after adding $30 \mathrm{mM}$ butyrate to a colon under low distention $\left(0 \mathrm{cmH}_{2} \mathrm{O}\right.$ outflow pressure) and $30 \mathrm{mM}$ propionate. (A) CMC frequency. (B) CMC amplitude. $\mathrm{N}=5, n=37$ where $\mathrm{N}$ indicates number of mice (separated by color and shape), $n$ indicates number of motor patterns (one data point). The ability for butyrate to increase the frequency of CMCs is inconsistent in the presence of propionate. In three mice (orange, green, pink), an increase in the number of observed CMCs corresponded to more data points after $30 \mathrm{mM}$ butyrate was added to propionate. In one mouse, no CMCs were observed, corresponding to no data points in Figure 8B. $P$ values (NS) were determined by a one-way ANOVA and Tukey's post hoc test.

of the length of the colon through 30 CMCs and seven peristaltic reflex movements. Specifics of these motor patterns are shown in Tables 2 and 3.

\section{Motor Patterns Associated With Pellet Expulsion With Krebs Perfusion From the Distal End in the Absence of Butyrate}

In 17 mice, 27 pellets moved out of the colon within $7.51 \pm 0.92 \mathrm{~min}$, through $26 \mathrm{CMCs}$ and 50 reflex movements (Figures 12A-D and Tables 2, 3). The CMC frequency did not differ upon insertion of the pellets, but the frequency of reflex movements increased from $0.06(0.04,0.12)$ to $0.25 \mathrm{cpm}$ $(0.13,0.74)(p=0.001)$ (Table 3 and Figure 12D). CMCs which moved these pellets were also of higher amplitude compared to those either before or after pellet insertion (Figure 12D). Finally, introducing distention via Krebs perfusion significantly increased the velocity of the reflex movements ( $p=0.012$, Table 2 ).

\section{Motor Patterns Associated With Pellet Expulsion With Butyrate}

The effect of butyrate on pellet expulsion was studied in colons with Krebs perfusion. Figure 14 shows changes in the peristaltic reflex movements in the presence of butyrate. When perfusion consisted solely of Krebs solution, 16 out of 21 pellets moved out of the colon in $30 \mathrm{~min}$, compared to 21 out of 23 pellets with $10 \mathrm{mM}$ of butyrate (Table 4). $3.06 \pm 0.27$ movements over $8.73 \pm 1.00 \mathrm{~min}$ were required to expel each pellet with Krebs perfusion, whereas with butyrate it took $2.67 \pm 0.28$ movements over $5.62 \pm 0.85 \mathrm{~min}(p=0.043$, Table 5). In the control group, 21 pellets moved out of the colon by 17 CMCs and 32 peristaltic reflexes, whereas with butyrate, 23 pellets moved out of the colon by $20 \mathrm{CMCs}$ and 36 peristaltic reflexes (Table 4). The average velocity of peristaltic reflex movements did not differ between these two phases $(0.30 \pm 0.04 \mathrm{~mm} / \mathrm{s}$ with Krebs and $0.38 \pm 0.04 \mathrm{~mm} / \mathrm{s}$ with $10 \mathrm{mM}$ butyrate) (Table 4). Similar to the effect of Krebs perfusion, the reflex movements were augmented with an increase in frequency from 0.19 to $0.46 \mathrm{cpm}$ by the introduction of butyrate $(p=0.036, N=10, n=23)$, while the frequency of CMCs remained similar.

\section{DISCUSSION}

Butyrate and propionate are primary SCFAs produced through the fermentation of indigestible carbohydrates, produced by specific subspecies of gut microbiota (Louis et al., 2016; Louis and Flint, 2017). These SCFAs are present in relative ratios of $1: 1$ in the human colon and have a potential role as signaling molecules that modulate colonic peristalsis (Hurst et al., 2014; Louis et al., 2016). Reigstad et al. (2015) demonstrated that enterochromaffin (EC) cells exposed to butyrate upregulate tryptophan hydroxylase-1 (Tph1), which catalyzes the formation of 5-HT, see also Martin et al. (2017). Although EC-released 5$\mathrm{HT}$ is not essential for the generation of CMCs (Keating and Spencer, 2010; Vincent et al., 2018), experimental data do support a physiological role for this process (Smith et al., 2014). We sought to better understand the role of butyrate in regulation of colonic peristalsis and the present study found that although mechanical distention is by far the most powerful stimulus for CMC generation, butyrate has a modulatory excitatory role under all conditions studied.

\section{CMCs Are Intrinsically Present Under Minimal Mechanical Distention}

Abundant literature exists describing colonic motor patterns in rabbits, guinea pigs, and rats, but those in the mouse remain relatively undetermined (Lentle et al., 2008; Chen et al., 2013; Costa et al., 2013; Shokrollahi et al., 2019). We first observed the mouse colon under baseline conditions to establish intrinsic motor patterns. Without stretch, distention or perfusion, proximal rhythmic contractions were often present, but were not classified as CMCs since they were 


\section{A}

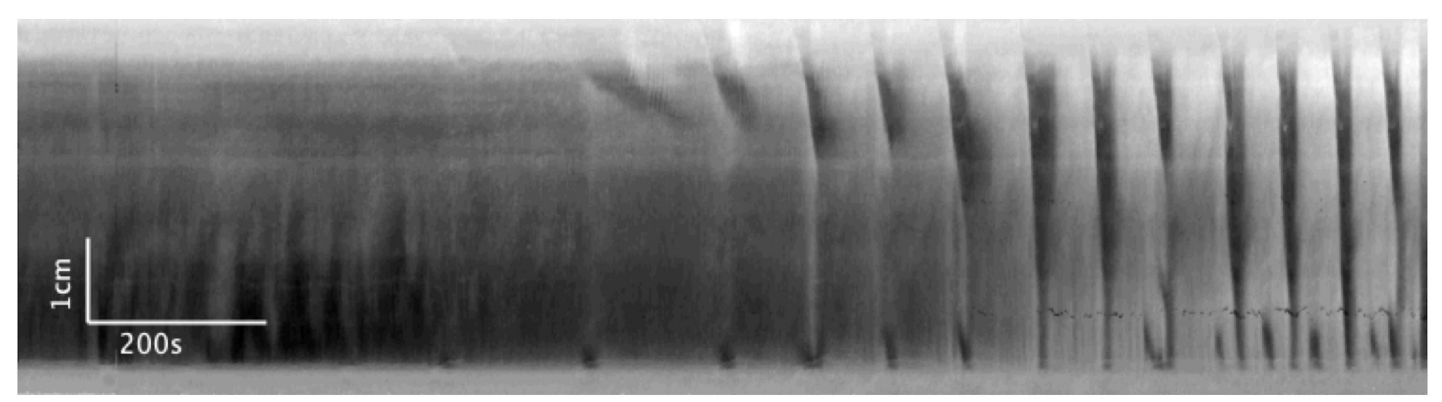

B

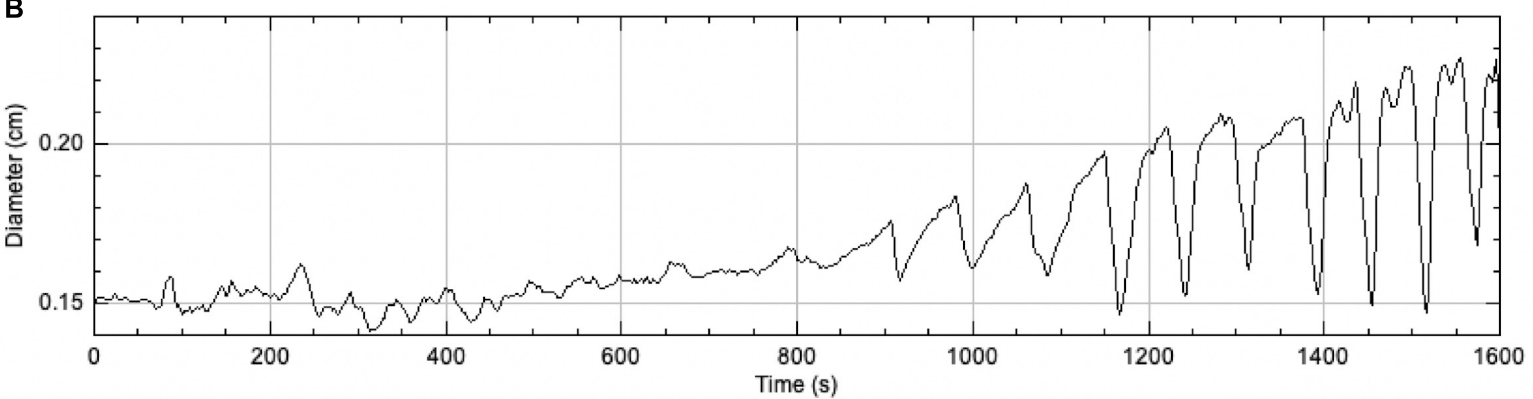

FIGURE 9 | Colonic activity in response to increasing distention in the presence of $30 \mathrm{mM}$ propionate. (A) Spatiotemporal map. (B) Average diameter over time. Distention is a strong stimulus which can easily overcome the inhibitory effects of propionate to generate strong LDCs.

not pan-colonic. In addition, low-amplitude CMCs in the form of clustered FPCs often occurred at a low cluster frequency, also shown in the rabbit colon (Chen et al., 2016). The presence of myogenic ripples indicates that ICC in the submuscular plexus (ICC-SMP) are also intrinsically active, with a hypothesized role in intestinal absorption (Huizinga et al., 2011).

Although CMCs are usually dependent on the presence of some intraluminal pressure, they can also occur in an empty colon without any intraluminal pressure as we show in the present study, albeit infrequently, under many experimental low distention conditions (Barnes et al., 2014). In this respect, the generation of a murine CMC is similar to that of human HighAmplitude Propagating Pressure Waves which occur without any apparent stimulus during High Resolution Colonic Motility when the colon is empty (Chen et al., 2017, 2018).

\section{Mechanical Distention Is a Strong Inducer of CMCs}

As Shokrollahi et al. (2019) reported in rabbits, we observed that CMCs in the mouse colon progress in predictable phases depending on the level of excitation (Figure 2). Individual colons may begin with varying baseline activities along this spectrum, but mechanical distention will almost always increase CMC frequency, propagation distance and amplitude (Huizinga et al., 2011; Spencer et al., 2016). First, minimal distention enhances the amplitude and frequency of ICC-MP orchestrated FPCs, indicating that they are usually present but attenuated in the background (Figure 2A; Alberti et al., 2005). Upon further excitation, clusters of FPCs can merge to create high-amplitude LDCs, the highest level of CMC activity (Figures 2C,D).
A strong physiological stimulus in the human colon is general distention caused by digested food or feces. In the mouse, we mimicked this by using perfusion-generated gradual increases in intraluminal pressure. However, increases in intraluminal pressure do not correlate linearly with changes in distention, as our experiments show that adaptive relaxation minimizes increases in pressure as well as stretch. When adaptive relaxation is overcome, pressure and/or stretch become powerful stimuli for the generation of CMCs. This general stretch evokes rhythmic motor patterns which differ from those caused by localized stretch. Similar to the guinea pig ileum (Spencer et al., 1999), the "law of the intestine" is unlikely to be responsible for the rhythmic propulsive motor complexes in the colon (Huizinga et al., 2011). CMCs are generated instead by myenteric neural activity superimposed on ICC-MP generated slow waves (Pluja et al., 2001; Yoneda et al., 2004). In extreme cases it was found that CMCs can prevail without the submucosal plexus and mucosa, but not without the myenteric plexus and muscularis externa (Zagorodnyuk and Spencer, 2011). Nevertheless, under standard physiological conditions, luminal stretch involves both neuronal and muscular components by potentially activating mucosal sensory neurons to evoke activity in the myenteric plexus (Smith et al., 2014), although no direct electrophysiological recordings from sensory nerves in the mouse colon have been obtained yet.

\section{Butyrate Augments CMCs in Low Activity Conditions}

Luminal butyrate at relatively low concentrations (1 $\mathrm{mM})$ increased CMC frequencies when distention-induced activity was low, or when the outflow pressure was $0 \mathrm{cmH}_{2} \mathrm{O}$. When CMC activity was pronounced with higher levels of distention, such as 


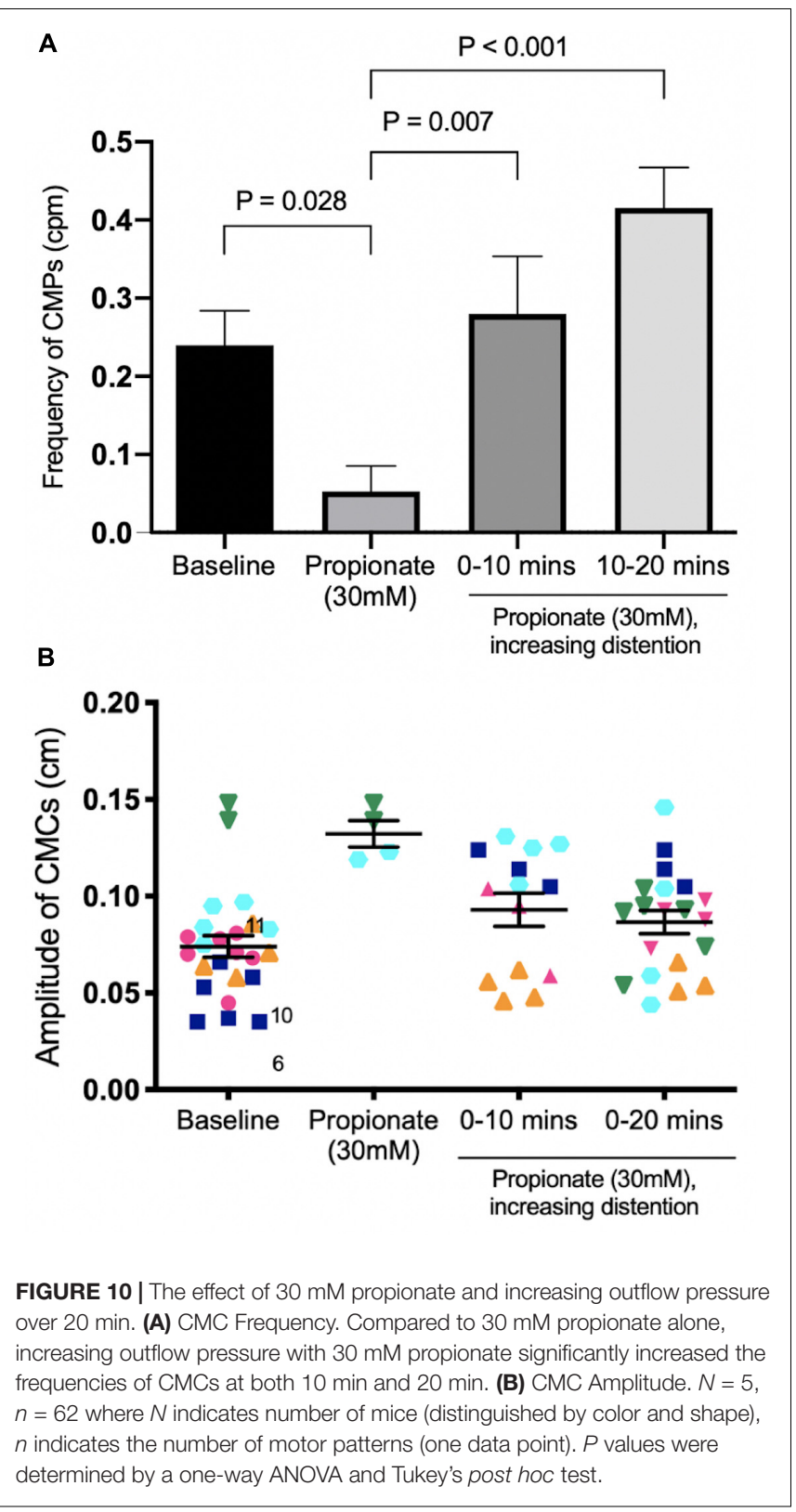

at with $3 \mathrm{cmH}_{2} \mathrm{O}$ outlflow pressure or when the outflow pressure was gradually increased, butyrate had no additional effects. Thus, it may be that butyrate does not have a significant role under normal physiological conditions, but its role might increase under conditions of low activity such as poor fiber intake where mechanical stimulation in the lumen is low. This butyrate action may occur by stimulating 5-HT production in enteroendocrine cells, which may act primarily on $5-\mathrm{HT}_{4}$ receptors on sensory nerves (Hoffman et al., 2012; Yu et al., 2015; Reigstad et al., 2016).

\section{Butyrate Has Variable Effects on CMC Activity in the Presence of Propionate}

Previous studies have postulated that propionate has an inhibitory effect on both short and full propagating contractions

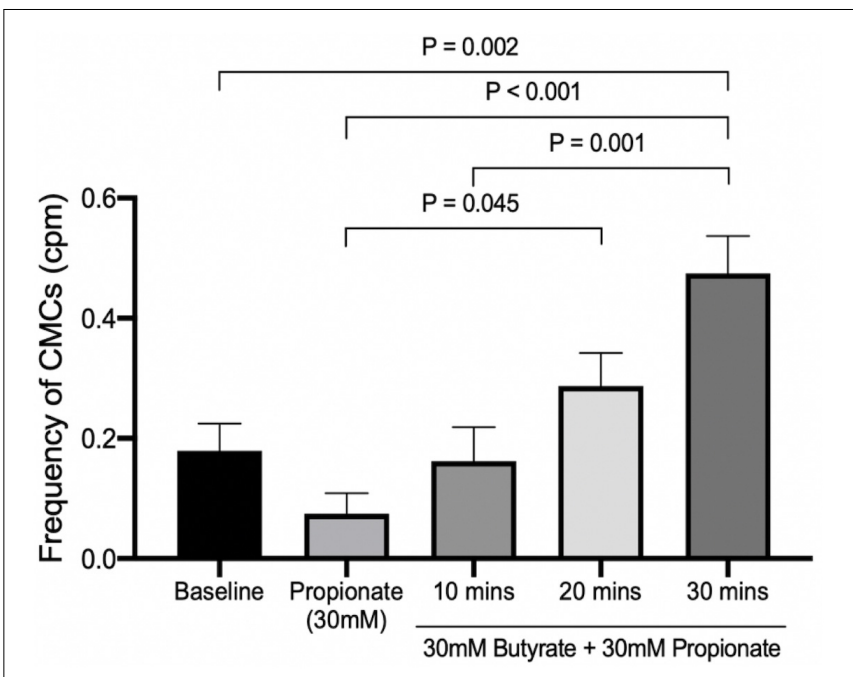

FIGURE 11 | The effect of $30 \mathrm{mM}$ butyrate on increasing mechanical distention's ability to regenerate CMCs under $30 \mathrm{mM}$ propionate. Since CMC frequencies increase in a pattern similar to that of Figure 10, the significant differences are more likely due to mechanical distention than the effects of butyrate. $P$ values were determined by a one-way ANOVA and Tukey's post hoc test.

(Tazoe et al., 2008). In the present study, propionate was a weak inhibitor and any inhibition could be easily overcome by minor distention (Figure 9). Interestingly, the average frequencies of CMCs sometimes remained constant or increased after the administration of propionate, resulting in low-amplitude FPCs of moderate frequency. This corresponds to findings by Tazoe et al. (2008) who reported that $1-5 \mathrm{mM}$ propionate could significantly increase the frequencies of "giant contractions" while decreasing their amplitudes by up to $400 \%$.

Throughout our experiments, the effect of butyrate was characterized by an inconsistent capacity to overcome propionate's inhibitory effects. Propionate and butyrate are endogenous ligands for two G-coupled protein receptors, FFAR2/GPR43 and FFAR3/GPR41. These receptors have been detected on enteroendocrine L cells throughout the GI tract, but a majority reside within the distal ileum and large intestine (Samuel et al., 2008). GPR41 couples with $\mathrm{G}_{i / o}$, is equally bound by propionate and butyrate, and has been shown to release peptide YY (PYY), thus playing a key role in lipolysis (Kaemmerer et al., 2010; Hurst et al., 2014). On the other hand, GPR43 is coupled to both $G_{q}$ and $G_{i / o}$ proteins and is preferentially bound by propionate over butyrate (Hurst et al., 2014). In addition to releasing PYY, this receptor is also involved in the downstream activation of mucosal mast cells and has been hypothesized to be involved in motility disorders associated with 5-HT signaling (Kaemmerer et al., 2010). The inhibitory effects of luminally administered propionate are accounted for by its activation of GPR41 and GPR43 to release PYY, an established satiety hormone that inhibits peristalsis and transit time. However, our experiments found that the capacity for butyrate to restore peristaltic activity to a propionate-infused colon was extremely variable, which indicates that these two 

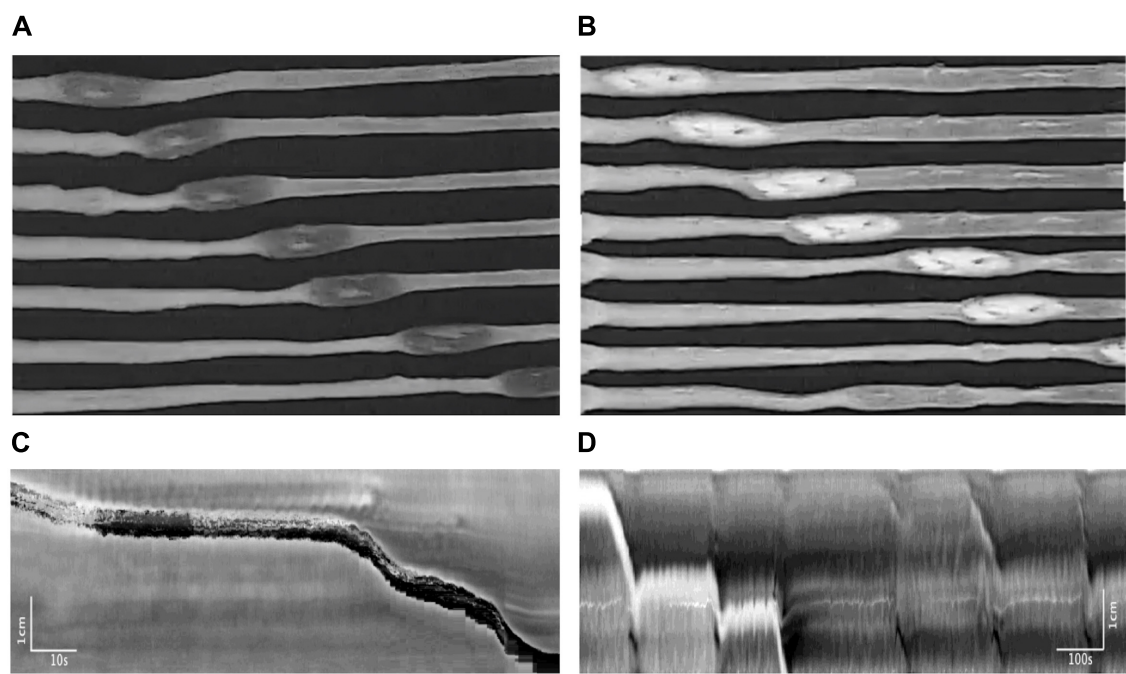

D

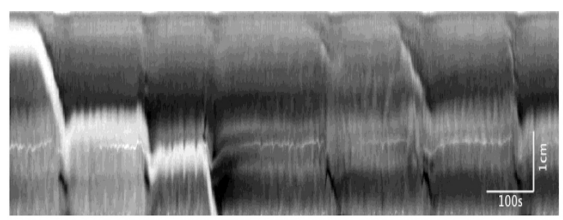

FIGURE 12 | Motor patterns associated with pellet expulsion with Krebs perfusion. (A) Movement of the pellet into the distal colon by the peristaltic reflex.

(B) Movement of the pellet into the distal colon by regularly occurring CMC. After traveling a partial length of the colon, the pellet may stop at one point for several minutes. It is eventually dislodged by intrinsic CMCs, which allow the pellet to become expelled at the anal end. Throughout this process, the frequencies of these CMCs do not change. (C) Spatiotemporal map of (A). (D) Spatiotemporal map of (B).

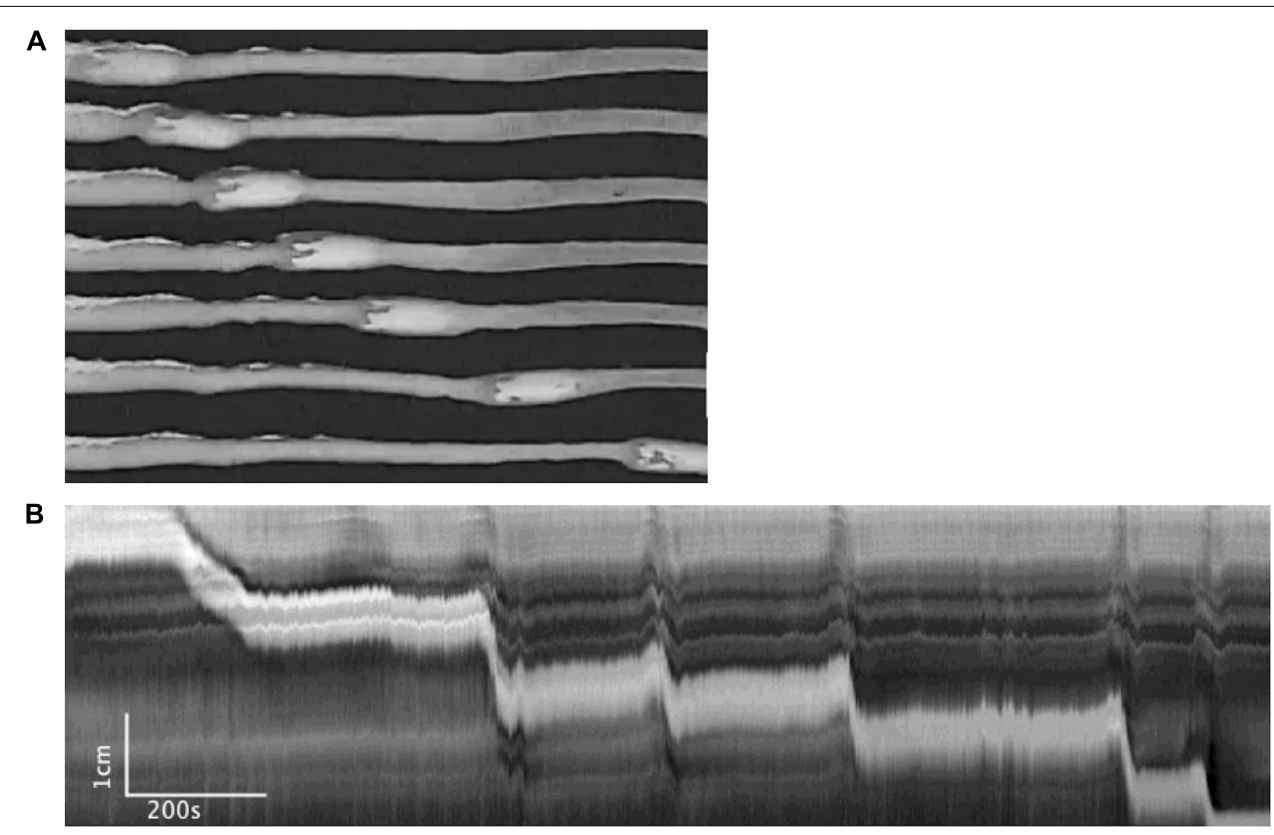

FIGURE 13 | Motor patterns associated with pellet expulsion without Krebs perfusion. (A) Distention-mediated reflex movements propel the pellet partially through the colon, before intrinsic neurogenic CMCs eventually expel it at the anal end. (B) Spatiotemporal maps associated with (A).

molecules act through separate pathways on enteroendocrine cells, and not necessarily through GPR receptors. Another target for butyrate is the Olf558 receptor, which has been colocalized to 5-HT-containing enteroendocrine cells (Bellono et al., 2017; Vincent et al., 2018). Since butyrate exists in equivalent concentrations and acts with a similar affinity toward this receptor, it may serve as a competitive antagonist to propionate on GPR43s. A decrease in PYY due to altered G protein coupling could be enough to lessen propionate's inhibitory effects, even if 5-HT was not directly released by the binding of butyrate. Thus, the complicated interactions between these two SCFAs may be explained by (a) competitiveness for GPR receptors and (b) two different activating receptors on enteroendocrine cells, which ultimately release separate signaling molecules to act on sensory neurons in the myenteric plexus. Overall, the strongest inducer of CMCs in all conditions was mechanical distention. 


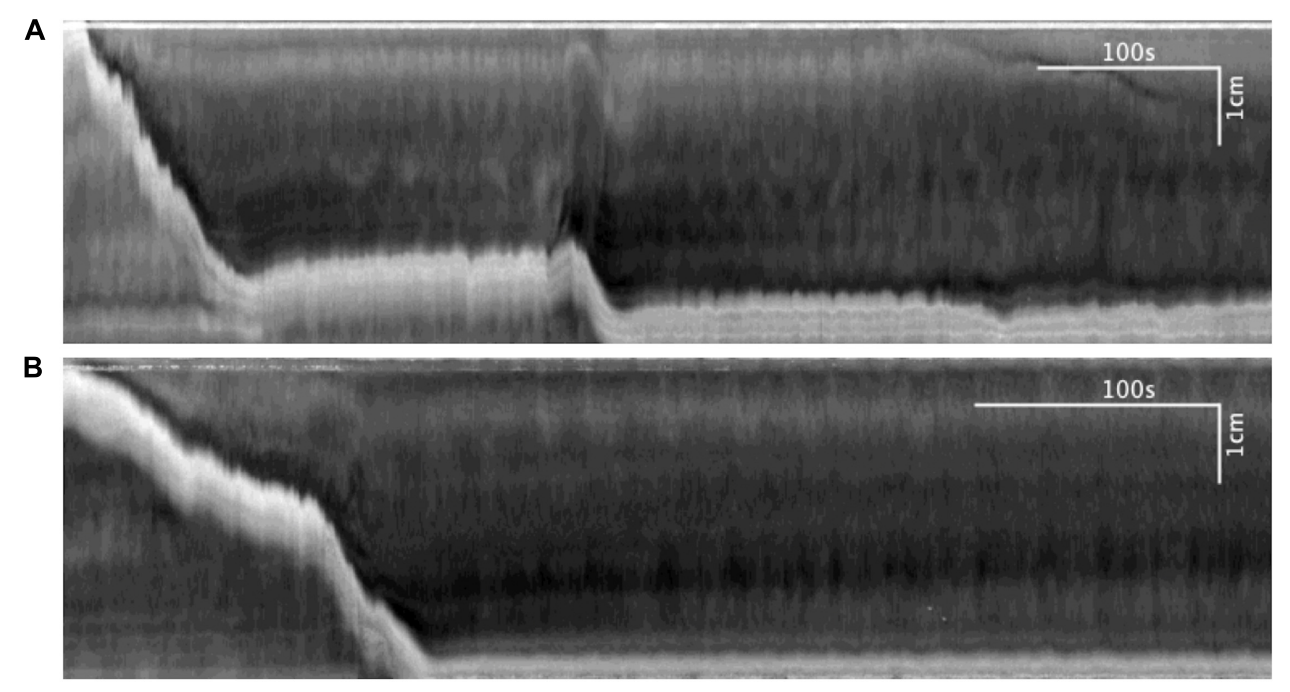

FIGURE 14 | (A) Spatiotemporal map of control. (B) Spatiotemporal map of $30 \mathrm{mM}$ butyrate on pellet propulsion.

\section{Butyrate Augments Two Types of Motor Activity Associated With Pellet Propulsion}

In the present study, pellets were propelled distally by two distinct mechanisms. One was the CMC; although not initiated by pellets, a CMC was able to move a pellet distally and 2-5 CMCs could expel the pellet from the colon. This intermittent propulsion by CMCs was also observed in the guinea pig colon (Costa et al., 2019). Weak CMCs increased in amplitude upon reaching the portion of the colon that was distended by the pellet (Figure 14). Pellets usually moved slightly orally caused by the relaxation phase of the CMCs. Similar to its effects with general distention, $10 \mathrm{mM}$ butyrate had an excitatory but inconsistent effect on the frequency of CMCs.

Another distinct motor pattern appeared only after pellet insertion, which was the peristaltic reflex initated by a pellet. A reflex often started with circular muscle contraction just oral to the pellet which then moved downward over a prolonged period of time. Unlike CMCs, they were caused directly by localized distention and are explained by the "law of the intestine": the peristaltic reflex seems to function via Bayliss and Starling's principles where the polarization of intrinsic neural pathways results in oral contraction and anal relaxation upon enteric mechano-sensory neural excitation (Bayliss and Starling, 1900; Costa and Furness, 1976). This motor pattern is a reflex in that it is initiated by pellet-induced stretching but is a complex process influenced by bolus size and pellet consistency (Costa et al., 2017). Furthermore, the reflex is influenced by luminal factors: the present study shows that both Krebs perfusion and $10 \mathrm{mM}$ butyrate greatly augmented the peristaltic reflexes generated.

Overall, localized distention did not seem to diminish butyrate's stimulatory capacity to the same degree as general distention. Butyrate augmented both CMCs and peristaltic reflexes to decrease the amount of time required to expel each pellet. Additionally, fewer movements were required to expel each pellet, indicating better coordination of motility patterns to increase the effectiveness of peristalsis.

\section{DATA AVAILABILITY STATEMENT}

The datasets generated for this study are available on request to the corresponding author.

\section{ETHICS STATEMENT}

The animal study was reviewed and approved by the McMaster University Animal Research Ethics Board.

\section{AUTHOR CONTRIBUTIONS}

$\mathrm{JH}$ and J-HC designed the study and supervised execution and analysis. WT did most of the experimentation and analysis and wrote the first draft of manuscript. GL conducted the propionate experiments and made a substantial contribution to analysis and manuscript writing. All authors approved the final version.

\section{FUNDING}

This study was supported by an operating grant from the Canadian Institutes of Health Research PJT-152942, and conducted at McMaster University. WT was supported by the National Natural Science Foundation of China (NSFC 81600423), the Chinese Scholarship Council, and the Farncombe Family Digestive Health Research Institute. GL was supported by an NSERC summer scholarship. 


\section{REFERENCES}

Alberti, E., Mikkelsen, H. B., Larsen, J. O., and Jimenez, M. (2005). Motility patterns and distribution of interstitial cells of Cajal and nitrergic neurons in the proximal, mid- and distal-colon of the rat. Neurogastroenterol. Motil. 17, 133-147. doi: 10.1111/j.1365-2982.2004.00603.x

Barnes, K. J., Beckett, E. A., Brookes, S. J., Sia, T. C., and Spencer, N. J. (2014). Control of intrinsic pacemaker frequency and velocity of colonic migrating motor complexes in mouse. Front. Neurosci. 8:96. doi: 10.3389/fnins.2014. 00096

Bayguinov, P. O., Hennig, G. W., and Smith, T. K. (2010). Ca2+ imaging of activity in ICC-MY during local mucosal reflexes and the colonic migrating motor complex in the murine large intestine. J. Physiol. 588, 4453-4474. doi: 10.1113/jphysiol.2010.196824

Bayliss, W. M., and Starling, E. H. (1900). The movements and the innervation of the large intestine. J. Physiol. 26, 107-118. doi: 10.1113/jphysiol.1900.sp000825

Beck, K., Voussen, B., Reigl, A., Vincent, A. D., Parsons, S. P., Huizinga, J. D., et al. (2019). Cell-specific effects of nitric oxide on the efficiency and frequency of long distance contractions in murine colon. Neurogastroenterol. Motil. 31:e13589. doi: 10.1111/nmo.13589

Bellono, N. W., Bayrer, J. R., Leitch, D. B., Castro, J., Zhang, C., O’Donnell, T. A., et al. (2017). Enterochromaffin cells are gut chemosensors that couple to sensory neural pathways. Cell 170, 185.e16-198.e16. doi: 10.1016/j.cell.2017. 05.034

Bornstein, J. C., Costa, M., and Grider, J. R. (2004). Enteric motor and interneuronal circuits controlling motility. Neurogastroenterol. Motil. 16(Suppl. 1), 34-38. doi: 10.1111/j.1743-3150.2004.00472.x

Chen, J.-H., Parsons, S. P., Shokrollahi, M., Wan, A., Vincent, A. D., Yuan, Y., et al. (2018). Characterization of simultaneous pressure waves as biomarkers for colonic motility assessed by high-resolution colonic manometry. Front. Physiol. 9:1248. doi: 10.3389/fphys.2018.01248

Chen, J.-H., Yang, Z., Yu, Y., and Huizinga, J. D. (2016). Haustral boundary contractions in the proximal 3-taeniated colon of the rabbit. Am. J. Physiol. 310, G181-G192.

Chen, J.-H., Yu, Y., Yang, Z., Yu, W.-Z., Chen, W. L., Kim, M. J. M., et al. (2017). Intraluminal pressure patterns in the human colon assessed by high-resolution manometry. Sci. Rep. 7:41436. doi: 10.1038/srep41436

Chen, J. H., Zhang, Q., Yu, Y., Li, K., Liao, H., Jiang, L. S., et al. (2013). Neurogenic and myogenic properties of pan-colonic motor patterns and their spatiotemporal organization in rats. PLoS One 8:e60474. doi: 10.3389/fphys. 2015.00205

Corsetti, M., Costa, M., Bassotti, G., Bharucha, A. E., Borelli, O., Dinning, P., et al. (2019). Translational consensus on terminology and definition of colonic motility by means of manometric and non-manometric techniques. Nat. Rev. Gastroenterol. Hepatol. 16, 559-579.

Corsetti, M., Pagliaro, G., Demedts, I., Deloose, E., Gevers, A., Scheerens, C., et al. (2017). Pan-colonic pressurizations associated with relaxation of the anal sphincter in health and disease: a new colonic motor pattern identified using high-resolution manometry. Am. J. Gastroenterol. 112, 479-489. doi: 10.1038/ ajg.2016.341

Costa, M., Dodds, K. N., Wiklendt, L., Spencer, N. J., Brookes, S. J., and Dinning, P. G. (2013). Neurogenic and myogenic motor activity in the colon of the guinea-pig, mouse, rabbit and rat. Am. J. Physiol. Gastrointest Liver Physiol. 305, G749-G759. doi: 10.1152/ajpgi.00227.2013

Costa, M., and Furness, J. B. (1976). The peristaltic reflex: an analysis of the nerve pathways and their pharmacology. Naunyn Schmiedebergs Arch. Pharmacol. 294, 47-60. doi: 10.1007/bf00692784

Costa, M., Keightley, L. J., Wiklendt, L., Hibberd, T. J., Arkwright, J. W., Omari, T., et al. (2019). Roles of three distinct neurogenic motor patterns during pellet propulsion in guinea-pig distal colon. J. Physiol. 597, 5125-5140. doi: 10.1113/ JP278284

Costa, M., Wiklendt, L., Keightley, L., Brookes, S. J. H., Dinning, P. G., and Spencer, N. J. (2017). New insights into neurogenic cyclic motor activity in the isolated guinea-pig colon. Neurogastroenterol. Motil. 29, 1-13. doi: 10.1111/nmo.13092

Costa, M., Wiklendt, L., Simpson, P., Spencer, N. J., Brookes, S. J., and Dinning, P. G. (2015). Neuromechanical factors involved in the formation and propulsion of fecal pellets in the guinea-pig colon. Neurogastroenterol. Motil. 27, 1466-1477. doi: 10.1111/nmo.12646
Dickson, E. J., Hennig, G. W., Heredia, D. J., Lee, H. T., Bayguinov, P. O., Spencer, N. J., et al. (2008). Polarized intrinsic neural reflexes in response to colonic elongation. J. Physiol. 586, 4225-4240. doi: 10.1113/jphysiol.2008.155630

Dinning, P. G. (2016). Recording in vivo human colonic motility: what have we learnt over the past 100 years. Adv. Exp. Med. Biol. 891, 213-222. doi: 10.1007/ 978-3-319-27592-5_20

Dinning, P. G., Wiklendt, L., Omari, T., Arkwright, J. W., Spencer, N. J., Brookes, S. J., et al. (2014). Neural mechanisms of peristalsis in the isolated rabbit distal colon: a neuromechanical loop hypothesis. Front. Neurosci. 8:75. doi: 10.3389/ fnins. 2014.00075

Fida, R., Lyster, D. J., Bywater, R. A., and Taylor, G. S. (1997). Colonic migrating motor complexes (CMMCs) in the isolated mouse colon. Neurogastroenterol. Motil. 9, 99-107. doi: 10.1046/j.1365-2982.1997.d01-25.x

Fukumoto, S., Tatewaki, M., Yamada, T., Fujimiya, M., Mantyh, C., Voss, M., et al. (2003). Short-chain fatty acids stimulate colonic transit via intraluminal 5-HT release in rats. Am. J. Physiol. Regul. Integr. Comp. Physiol. 284, R1269-R1276.

Hoffman, J. M., Tyler, K., MacEachern, S. J., Balemba, O. B., Johnson, A. C., Brooks, E. M., et al. (2012). Activation of colonic mucosal 5-HT(4) receptors accelerates propulsive motility and inhibits visceral hypersensitivity. Gastroenterology 142, 844.e-854.e. doi: 10.1053/j.gastro.2011.12.041

Huizinga, J. D., Martz, S., Gill, V., Wang, X.-Y., Jimenez, M., and Parsons, S. (2011). Two independent networks of interstitial cells of Cajal work cooperatively with the enteric nervous system to create colonic motor patterns. Front. Neurosci. 5:93. doi: $10.3389 /$ fnins.2011.00093

Hurst, N. R., Kendig, D. M., Murthy, K. S., and Grider, J. R. (2014). The short chain fatty acids, butyrate and propionate, have differential effects on the motility of the guinea pig colon. Neurogastroenterol. Motil. 26, 1586-1596. doi: 10.1111/ nmo. 12425

Kaemmerer, E., Plum, P., Klaus, C., Weiskirchen, R., Liedtke, C., Adolf, M., et al. (2010). Fatty acid binding receptors in intestinal physiology and pathophysiology. World J. Gastrointest. Pathophysiol. 1, 147-153.

Keating, D. J., and Spencer, N. J. (2010). Release of 5-hydroxytryptamine from the mucosa is not required for the generation or propagation of colonic migrating motor complexes. Gastroenterology 138, 659-70670.e1. doi: 10.1053/j.gastro. 2009.09.020

Kendig, D. M., and Grider, J. R. (2015). Serotonin and colonic motility. Neurogastroenterol. Motil. 27, 899-905. doi: 10.1111/nmo.12617

Kendig, D. M., Hurst, N. R., Bradley, Z. L., Mahavadi, S., Kuemmerle, J. F., Lyall, V., et al. (2014). Activation of the umami taste receptor (T1R1/T1R3) initiates the peristaltic reflex and pellet propulsion in the distal colon. Am. J. Physiol. Gastrointest. Liver Physiol. 307, G1100-G1107. doi: 10.1152/ajpgi.00251.2014

Lentle, R. G., Janssen, P. W., Asvarujanon, P., Chambers, P., Stafford, K. J., and Hemar, Y. (2008). High-definition spatiotemporal mapping of contractile activity in the isolated proximal colon of the rabbit. J. Comp. Physiol. B 178, 257-268. doi: 10.1007/s00360-007-0217-9

Li, H., Chen, J. H., Yang, Z., Huang, M., Yu, Y., Tan, S., et al. (2016). Neurotensin changes propulsive activity into a segmental motor pattern in the rat colon. J. Neurogastroenterol. Motil. 22, 517-528. doi: 10.5056/jnm15181

Louis, P., and Flint, H. J. (2017). Formation of propionate and butyrate by the human colonic microbiota. Environ. Microbiol. 19, 29-41. doi: 10.1111/14622920.13589

Louis, P., Flint, H. J., and Michel, C. (2016). How to manipulate the microbiota: prebiotics. Adv. Exp. Med. Biol. 902, 119-142. doi: 10.1007/978-3-319-312484_9

Martin, A. M., Lumsden, A. L., Young, R. L., Jessup, C. F., Spencer, N. J., and Keating, D. J. (2017). Regional differences in nutrient-induced secretion of gut serotonin. Physiol. Rep. 5:e13199. doi: 10.14814/phy2.13199

Parsons, S. P., and Huizinga, J. D. (2015). Effects of gap junction inhibition on contraction waves in the murine small intestine in relation to coupled oscillator theory. Am. J. Physiol. Gastrointest. Liver Physiol. 308, G287-G297. doi: 10. 1152/ajpgi.00338.2014

Pluja, L., Alberti, E., Fernandez, E., Mikkelsen, H. B., Thuneberg, L., and Jimenez, M. (2001). Evidence supporting presence of two pacemakers in rat colon. Am. J. Physiol. Gastrointest. Liver Physiol. 281, G255-G266.

Powell, A. K., Fida, R., and Bywater, R. A. (2003). Motility in the isolated mouse colon: migrating motor complexes, myoelectric complexes and pressure waves. Neurogastroenterol. Motil. 15, 257-266. doi: 10.1046/j.1365-2982.2003. 00412.x 
Quan, X., Yang, Z., Xue, M., Chen, J.-H., and Huizinga, J. D. (2017). Relationships between motor patterns and intraluminal pressure in the 3-taeniated proximal colon of the rabbit. Nat. Sci. Rep. 7:42293. doi: 10.1038/srep42293

Reigstad, C. S., Linden, D. R., Szurszewski, J. H., Sonnenburg, J. L., Farrugia, G., and Kashyap, P. C. (2016). Correlated gene expression encoding serotonin (5$\mathrm{HT}$ ) receptor 4 and 5-HT transporter in proximal colonic segments of mice across different colonization states and sexes. Neurogastroenterol. Motil. 28, 1443-1448. doi: 10.1111/nmo.12840 doi: 10.1111/nmo.12840

Reigstad, C. S., Salmonson, C. E., Rainey, J. F., Szurszewski, J. H., Linden, D. R., Sonnenburg, J. L., et al. (2015). Gut microbes promote colonic serotonin production through an effect of short-chain fatty acids on enterochromaffin cells. FASEB J. 29, 1395-1403. doi: 10.1096/fj.14-259598

Roberts, R. R., Bornstein, J. C., Bergner, A. J., and Young, H. M. (2008). Disturbances of colonic motility in mouse models of Hirschsprung's disease. Am. J. Physiol. Gastrointest. Liver Physiol. 294, G996-G1008. doi: 10.1152/ajpgi. 00558.2007

Roberts, R. R., Murphy, J. F., Young, H. M., and Bornstein, J. C. (2007). Development of colonic motility in the neonatal mouse-studies using spatiotemporal maps. Am. J. Physiol. Gastrointest. Liver Physiol. 292, G930G938.

Samuel, B. S., Shaito, A., Motoike, T., Rey, F. E., Backhed, F., Manchester, J. K., et al. (2008). Effects of the gut microbiota on host adiposity are modulated by the short-chain fatty-acid binding G protein-coupled receptor, Gpr41. Proc. Natl. Acad. Sci. U.S.A. 105, 16767-16772. doi: 10.1073/pnas.0808567105

Shokrollahi, M., Chen, J. H., and Huizinga, J. D. (2019). Intraluminal prucalopride increases propulsive motor activities via luminal 5-HT4 receptors in the rabbit colon. Neurogastroenterol. Motil. 31:e13598. doi: 10.1111/nmo.13598

Sia, T. C., Brookes, S. J., Dinning, P. G., Wattchow, D. A., and Spencer, N. J. (2013). Peristalsis and propulsion of colonic content can occur after blockade of major neuroneuronal and neuromuscular transmitters in isolated guinea pig colon. Am. J. Physiol. Gastrointest. Liver Physiol. 305, G933-G939. doi: 10.1152/ajpgi.00257.2013

Smith, T. K., Oliver, G. R., Hennig, G. W., O’Shea, D. M., Vanden Berghe, P., Kang, S. H., et al. (2003). A smooth muscle tone-dependent stretch-activated migrating motor pattern in isolated guinea-pig distal colon. J. Physiol. 551, 955-969. doi: 10.1113/jphysiol.2003.049163

Smith, T. K., Park, K. J., and Hennig, G. W. (2014). Colonic migrating motor complexes, high amplitude propagating contractions, neural reflexes and the importance of neuronal and mucosal serotonin. J. Neurogastroenterol. Motil. 20, 423-446. doi: 10.5056/jnm14092

Spencer, N., Walsh, M., and Smith, T. K. (1999). Does the guinea-pig ileum obey the 'law of the intestine'? J. Physiol. 517, 889-898. doi: 10.1111/j.1469-7793. 1999.0889s.x
Spencer, N. J., Dinning, P. G., Brookes, S. J., and Costa, M. (2016). Insights into the mechanisms underlying colonic motor patterns. J. Physiol. 594, 4099-4116. doi: 10.1113/JP271919

Spencer, N. J., Hennig, G. W., and Smith, T. K. (2002). A rhythmic motor pattern activated by circumferential stretch in guinea-pig distal colon. J. Physiol. 545, 629-648. doi: 10.1113/jphysiol.2002.028647

Spencer, N. J., Travis, L., Wiklendt, L., Hibberd, T. J., Costa, M. C., Dinning, P., et al. (2019). Diversity of neurogenic smooth muscle electrical rhythmicity in mouse proximal colon. Am. J. Physiol. Gastrointest. Liver Physiol. 318, G244-G253. doi: 10.1152/ajpgi.00317.2019

Tazoe, H., Otomo, Y., Kaji, I., Tanaka, R., Karaki, S. I., and Kuwahara, A. (2008). Roles of short-chain fatty acids receptors, GPR41 and GPR43 on colonic functions. J. Physiol. Pharmacol. 59(Suppl. 2), 251-262.

Vincent, A. D., Wang, X. Y., Parsons, S. P., Khan, W. I., and Huizinga, J. D. (2018). Abnormal absorptive colonic motor activity in germ free mice is rectified by butyrate, an effect possibly mediated by mucosal serotonin. Am. J. Physiol. 315, G896-G907. doi: 10.1152/ajpgi.00237.2017

Yoneda, S., Fukui, H., and Takaki, M. (2004). Pacemaker activity from submucosal interstitial cells of Cajal drives high-frequency and low-amplitude circular muscle contractions in the mouse proximal colon. Neurogastroenterol. Motil. 16, 621-627. doi: 10.1111/j.1365-2982.2004.00546.x

Yoneda, S., Takano, H., Takaki, M., and Suzuki, H. (2002). Properties of spontaneously active cells distributed in the submucosal layer of mouse proximal colon. J. Physiol. 542, 887-897. doi: 10.1113/jphysiol.2002.018705

Yu, Y., Chen, J. H., Li, H., Yang, Z., Du, X., Hong, L., et al. (2015). Involvement of 5HT3 and 5-HT4 receptors in colonic motor patterns in rats. Neurogastroenterol. Motil. 27, 914-928. doi: 10.1111/nmo.12550

Zagorodnyuk, V. P., and Spencer, N. J. (2011). Localization of the sensory neurons and mechanoreceptors required for stretch-evoked colonic migrating motor complexes in mouse colon. Front. Physiol. 2:98. doi: 10.3389/fphys.2011. 00098

Conflict of Interest: The authors declare that the research was conducted in the absence of any commercial or financial relationships that could be construed as a potential conflict of interest.

Copyright (c) 2020 Tan, Lee, Chen and Huizinga. This is an open-access article distributed under the terms of the Creative Commons Attribution License (CC BY). The use, distribution or reproduction in other forums is permitted, provided the original author(s) and the copyright owner(s) are credited and that the original publication in this journal is cited, in accordance with accepted academic practice. No use, distribution or reproduction is permitted which does not comply with these terms. 